\title{
Injection of large transverse emittance EBIS beams in booster
}

\author{
C.J. Gardner
}

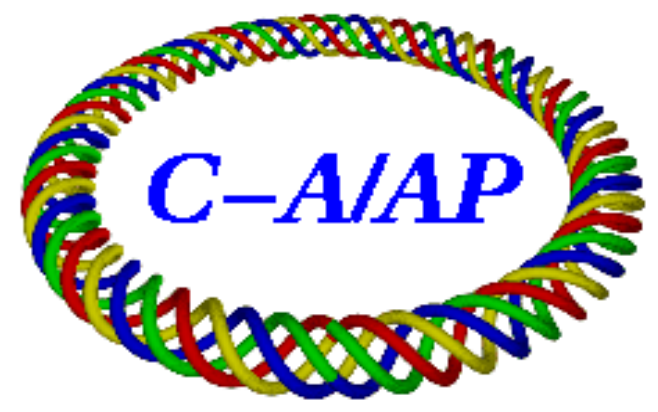

\section{Collider-Accelerator Department Brookhaven National Laboratory Upton, NY 11973}

Notice: This document has been authorized by employees of Brookhaven Science Associates, LLC under Contract No. DE-AC02-98CH10886 with the U.S. Department of En ergy. The United States Government retains a nonexclusive, paid-up, irrevocable, world-wide license to publish or reproduce the published form of this document, or allow others to do so, for United States Government purposes. 


\title{
Injection of Large Transverse Emittance EBIS Beams in Booster
}

\author{
C.J. Gardner
}

October 10, 2011

During the commissioning of EBIS beams in Booster in November 2010 and in April, May and June 2011, it was found that the transverse emittances of the EBIS beams just upstream of Booster were much larger than expected. Beam emittances of $11 \pi \mathrm{mm}$ milliradians had been expected, but numbers 3 to 4 times larger were measured.

Here and throughout this note the beam emittance, $\pi \epsilon_{0}$, is taken to be the area of the smallest ellipse that contains $95 \%$ of the beam. We call this smallest ellipse the beam ellipse. If the beam distribution is gaussian, the rms emittance of the distribution is very nearly one sixth the area of the beam ellipse. The normalized rms emittance is the rms emittance times the relativistic factor $\beta \gamma=0.06564$. This amounts to $0.12 \pi \mathrm{mm}$ milliradians for the $11 \pi \mathrm{mm}$ milliradian beam ellipse.

In [1] we modeled the injection and turn-by-turn evolution of an $11 \pi \mathrm{mm}$ milliradian beam ellipse in the horizontal plane in Booster. It was shown that with the present injection system, up to 4 turns of this beam could be injected and stored in Booster without loss. In the present note we extend this analysis to the injection of larger emittance beams.

We consider only the emittance in the horizontal plane. Emittance in the vertical plane and the effects of dispersion are treated in [2].

\section{Beam Ellipse Transport in the Inflector}

The Inflector is described in [3]. The nominal path length is

$$
L=2.37274 \text { meters }
$$


and the radius of curvature is

$$
\rho=8.74123 \text { meters }
$$

This gives turning angle

$$
\theta=L / \rho=15.5525 \text { degrees. }
$$

The horizontal half-aperture is

$$
a=8.5 \mathrm{~mm}
$$

Let $\alpha_{0}, \beta_{0}, \gamma_{0}$ be the Courant-Snyder parameters of the incoming beam ellipse at the inflector exit. Then the beam ellipse parameters at the inflector entrance (i.e. at the upstream end) are given by

$$
\begin{gathered}
\beta_{1}=M_{11}^{2} \beta_{0}-2 M_{11} M_{12} \alpha_{0}+M_{12}^{2} \gamma_{0} \\
\alpha_{1}=-M_{11} M_{21} \beta_{0}+\left(1+2 M_{12} M_{21}\right) \alpha_{0}-M_{12} M_{22} \gamma_{0} \\
\gamma_{1}=M_{21}^{2} \beta_{0}-2 M_{21} M_{22} \alpha_{0}+M_{22}^{2} \gamma_{0}
\end{gathered}
$$

where

$$
\begin{aligned}
& M_{11}=\cos \theta, \quad M_{12}=-\rho \sin \theta \\
& M_{21}=\frac{1}{\rho} \sin \theta, \quad M_{22}=\cos \theta .
\end{aligned}
$$

Here $M_{11}, M_{12}, M_{21}, M_{22}$ are the elements of the transfer matrix from the inflector exit backwards to the entrance.

\section{Matching to the Inflector Channel}

The horizontal acceptance of the inflector channel is

$$
\pi e=\frac{\pi a^{2}}{\beta_{M}}
$$

where $\beta_{M}$ is the maximum attained by

$$
\beta(\phi)=\beta_{0} \cos ^{2} \phi+2 \alpha_{0} \rho \cos \phi \sin \phi+\gamma_{0} \rho^{2} \sin ^{2} \phi
$$

in the interval $0 \leq \phi \leq \theta$. 
For $0<\theta<\pi / 2$, a necessary condition for maximum acceptance is that $\beta(\phi)$ have a local minimum at $\phi=\theta / 2$, i.e. half-way through the inflector channel. One then has

$$
\beta_{M}=\beta_{0}=\beta_{1}
$$

and the acceptance is

$$
\pi e=\frac{\pi a^{2}}{\beta_{0}} .
$$

The maximum acceptance occurs when $\beta_{0}$ is smallest. Substituting $\beta_{0}$ for $\beta_{1}$ in (5) and multiplying by $\beta_{0}$ we have

$$
\beta_{0}^{2}=\left(M_{11} \beta_{0}-M_{12} \alpha_{0}\right)^{2}+M_{12}^{2} .
$$

This shows that $\beta_{0}$ is smallest when

$$
M_{11} \beta_{0}-M_{12} \alpha_{0}=0 .
$$

This gives

$$
\beta_{0}=-M_{12}=\rho \sin \theta
$$

and

$$
\alpha_{0}=\frac{M_{11}}{M_{12}} \beta_{0}=-M_{11}=-\cos \theta .
$$

The maximum acceptance is then

$$
\pi e_{M}=\frac{\pi a^{2}}{\rho \sin \theta} .
$$

A beam ellipse with Courant-Snyder parameters given by (16) and (17) is said to be matched to the inflector channel.

Putting numbers from (2), (3) and (4) into (16), (17) and (18) we obtain

$$
\alpha_{0}=-0.9634, \quad \beta_{0}=2.344 \text { meters }
$$

and

$$
\pi e_{M}=30.8 \pi \mathrm{mm} \text { milliradians. }
$$

If the horizontal half-aperture $a$ is increased from 8.5 to $10.5 \mathrm{~mm}$, the maximum acceptance becomes

$$
\pi e_{M}=47.0 \pi \mathrm{mm} \text { milliradians. }
$$

Plots of the horizontal beam envelope $\sqrt{\epsilon_{0} \beta}$ are shown in Figure 1 for the case in which the beam ellipse is matched to the inflector channel. Here the violet, blue, orange, and red curves show the envelope in the channel for beam ellipse emittances $\pi \epsilon_{0}=11 \pi, 22 \pi, 33 \pi$, and $44 \pi \mathrm{mm}$ milliradians respectively. The green and brown curves show the envelope for $\pi \epsilon_{0}=30.8 \pi$ and $47.0 \pi \mathrm{mm}$ milliradians respectively. 


\section{Mismatch between Beam Ellipse and Lattice}

Let $\alpha$ and $\beta$ be the Courant-Snyder parameters of the Booster lattice at the inflector exit. An ellipse with these parameters is said to be matched to the lattice and is called the machine ellipse. The MAD code gives $\alpha=-1.736$ and $\beta=10.96$ meters. Define parameters

$$
A=\alpha_{0}-\alpha B, \quad B=\beta_{0} / \beta, \quad G=\left(1+A^{2}\right) / B
$$

and

$$
H=\frac{1}{2}(B+G) .
$$

Then the mismatch between the incoming beam ellipse and the machine ellipse is given by the factor $[4,5]$

$$
\epsilon / \epsilon_{0}=H+\sqrt{H^{2}-1}
$$

where $\pi \epsilon_{0}$ is the emittance of the beam ellipse and $\pi \epsilon$ is the emittance of the smallest machine ellipse that contains the beam ellipse. Using (22) in (23) we also have

$$
H=\frac{1}{2}\left(\gamma \beta_{0}-2 \alpha \alpha_{0}+\beta \gamma_{0}\right)
$$

For the case in which the beam ellipse is matched to the inflector channel we have ellipse parameters (19) which give

$$
A=-0.5922, \quad B=0.2138, \quad H=3.265
$$

and mismatch factor

$$
\epsilon / \epsilon_{0}=6.37 \text {. }
$$

This large mismatch factor makes inefficient use of the Booster acceptance and makes injection of the beam ellipse more difficult. This is illustrated in Figures 2 through 4.

Figure 2 shows the incoming beam ellipse and its relation to the machine ellipse. Here the large and small black ellipses are the machine and beam ellipses, respectively, at the inflector exit. These evolve into the red ellipses in the 0.64253 meter drift from the inflector exit to quadrupole QHC4 of the Booster lattice. The half-aperture of the quadrupole is $76 \mathrm{~mm}$. The beam ellipse has emittance $\pi \epsilon_{0}=11 \pi \mathrm{mm}$ milliradians and is matched to the inflector channel. It ends up safely inside the QHC4 quadrupole aperture. The black machine ellipse is centered on the Booster beam pipe 
and just touches the septum of the inflector. The outer side of the $1 \mathrm{~mm}$ thick septum is $48.5 \mathrm{~mm}$ from the center of the beam pipe. The solid brown ellipse is the incoming beam ellipse shifted to the center of the machine ellipse. The dashed brown ellipse is the smallest machine ellipse that contains the beam ellipse. Its emittance, $\pi \epsilon$, is given by the mismatch factor (27).

In Figure 3 the incoming beam ellipse emittance has been increased to $\pi \epsilon_{0}=22 \pi \mathrm{mm}$ milliradians. This ellipse again ends up safely inside the QHC4 quadrupole aperture at $76 \mathrm{~mm}$. The solid and dashed brown ellipses are still inside but getting close to the black machine ellipse border.

In Figure 4 the incoming beam ellipse emittance has been increased to $\pi \epsilon_{0}=33 \pi \mathrm{mm}$ milliradians. This ellipse ends up close to the QHC4 quadrupole aperture. The solid and dashed brown ellipses now extend a little beyond the black machine ellipse border.

The mismatched beam ellipse in these figures stays inside the dashed brown machine ellipse but can rotate turn-by-turn about the center of the dashed ellipse. As it does, the two points of contact it has with the machine ellipse move along the machine ellipse border and it stretches and contracts, all the while keeping its area the same. The the dashed brown ellipse therefore needs to be kept away from the inflector septum in order to keep the beam ellipse from hitting the septum. For beam ellipses with large emittance and large mismatch factor (27), this means that the injection bump needs to be collapsed more quickly away from the septum than it would for smaller beam ellipses and smaller mismatch factors. This makes efficient injection of large beam ellipses with large mismatch factors difficult (if not impossible) with the present injection system [1].

\section{Beam Ellipse Matched to Lattice}

On the other hand, for the case in which the beam ellipse is matched to the Booster lattice, we have

$$
\alpha_{0}=\alpha, \quad \beta_{0}=\beta
$$

which gives

$$
A=0, \quad B=1, \quad H=1
$$

and mismatch factor

$$
\epsilon / \epsilon_{0}=1 \text {. }
$$


In this case the acceptance of the inflector channel is

$$
\pi e=\frac{\pi a^{2}}{\beta} .
$$

Using half-aperture $a=8.5 \mathrm{~mm}$ and $\beta=10.96$ meters, this amounts to only $6.59 \mathrm{pi} \mathbf{m m}$ milliradians. If the half-aperture is increased to 10.5 $\mathrm{mm}$, the acceptance increases to $\mathbf{1 0 . 1} \mathbf{~ p i ~} \mathbf{~ m m}$ milliradians, which is still less than the nominal 11 pi mm milliradian beam ellipse emittance.

Horizontal beam envelopes in the inflector channel for the case in which the beam ellipse is matched to the Booster lattice are plotted in Figure 5. Here the blue, red and brown curves show the envelope for beam ellipse emittances $6 \pi, 8 \pi$ and $11 \pi \mathrm{mm}$ milliradians respectively.

Figures 6 through $\mathbf{8}$ show the incoming beam ellipse and its relation to the machine ellipse. The black ellipses are again the machine and incoming beam ellipses at the inflector exit. These evolve into the red ellipses in the drift to the QHC4 quadrupole. The half-aperture of the quadrupole is 76 $\mathrm{mm}$. The incoming beam ellipse is matched to the lattice. The brown ellipse is the incoming beam ellipse shifted to the center of the black machine ellipse.

In Figure 6 the beam ellipse has emittance $\pi \epsilon_{0}=6 \pi \mathrm{mm}$ milliradians. It ends up safely inside the QHC4 quadrupole aperture.

In Figure 7 the beam ellipse emittance has been increased to $8 \pi \mathrm{mm}$ milliradians. This ellipse ends up safely inside but close to the QHC4 quadrupole aperture.

In Figure 8 the beam ellipse emittance has been increased to $11 \pi \mathrm{mm}$ milliradians. This ellipse ends up scraping against the QHC4 quadrupole aperture.

The conclusion here is that a beam ellipse matched to the machine lattice makes inefficient use of the inflector aperture and the aperture in the QHC4 quadrupole.

\section{Compromise between Inflector and Lattice Matching}

We are therefore led to consider incoming beam ellipse parameters that are somewhere between those that are matched to the inflector channel and 
those that are matched to the machine lattice. These can be specified in terms of parameters $B$ and $A$. For any given $B$ the mismatch factor (24) is smallest when $A=0$. This gives

$$
H=\frac{1}{2}\left(B+\frac{1}{B}\right)
$$

and the mismatch factor becomes simply

$$
\epsilon / \epsilon_{0}=1 / B \text {. }
$$

We then also have

$$
\alpha_{0}=\alpha B, \quad \beta_{0}=\beta B
$$

and the necessary condition (14) for maximum acceptance in the inflector becomes

$$
\beta^{2} B^{2}=\left(M_{11} \beta-M_{12} \alpha\right)^{2} B^{2}+M_{12}^{2} .
$$

This gives

$$
B^{2}=M_{12}^{2}\left\{\beta^{2}-\left(M_{11} \beta-M_{12} \alpha\right)^{2}\right\}^{-1} .
$$

Putting in numbers we obtain

$$
B=0.26537, \quad \alpha_{0}=-0.461, \quad \beta_{0}=2.91 \text { meters }
$$

and mismatch factor

$$
\epsilon / \epsilon_{0}=3.77 \text {. }
$$

The maximum acceptance of the inflector is then

$$
\pi e_{M}=\frac{\pi a^{2}}{\beta B}=24.8 \pi \text { mm milliradians. }
$$

Here we have used half-aperture $a=8.5 \mathrm{~mm}$. If the half-aperture is increased to $10.5 \mathrm{~mm}$, the maximum acceptance becomes

$$
\pi e_{M}=37.9 \pi \mathrm{mm} \text { milliradians. }
$$

These numbers are less than the maximums given by (20) and (21), but the benefit is that the mismatch factor has been reduced from 6.37 to 3.77 . For $B \geq 0.26537$ (and $A=0$ ) the inflector acceptance is

$$
\pi e_{0}=\frac{\pi a^{2}}{\beta_{0}}
$$


where $\beta_{0}=\beta B$. This is shown by the red curve in Figure 9. Here the half-aperture is taken to be $a=8.5 \mathrm{~mm}$.

For $B \leq 0.26537$ the acceptance is

$$
\pi e_{1}=\frac{\pi a^{2}}{\beta_{1}}
$$

where

$$
\beta_{1}=\beta_{0} \cos ^{2} \theta+2 \alpha_{0} \rho \cos \theta \sin \theta+\gamma_{0} \rho^{2} \sin ^{2} \theta
$$

and

$$
\alpha_{0}=\alpha B, \quad \beta_{0}=\beta B, \quad \gamma_{0}=\left(1+\alpha_{0}^{2}\right) / \beta_{0} .
$$

This is shown by the blue curve. The two curves intersect at $B=0.26537$ which gives the maximum acceptance.

Plots of the inflector acceptance versus $B$ for various values of the half-aperture $a$ are shown in Figure 10. Here, as before, we have taken $A=0$. The red, orange, green, blue, and violet curves give the acceptance for horizontal half-apertures $a=8.5,9.5,10.5,11.5$ and $12.5 \mathrm{~mm}$ respectively. For each curve the maximum acceptance occurs at $B=0.26537$.

For the slightly higher $B=0.3$, the red curve in Figure $\mathbf{1 0}$ shows that the acceptance is $\mathbf{2 2 . 0} \mathbf{~ p i ~} \mathbf{~ m m}$ milliradians for half-aperture $a=8.5 \mathrm{~mm}$. If the half-aperture is increased to $10.5 \mathrm{~mm}$, the acceptance increases to $\mathbf{3 3 . 5}$ pi $\mathbf{~ m m}$ milliradians as shown by the green curve. These numbers are lower than those in (39) and (40), but the mismatch factor is reduced further to

$$
\epsilon / \epsilon_{0}=1 / B=3.33 \text {. }
$$

We shall take $B=0.3$ (and $A=0$ ) as the compromise between matching to the inflector and matching to the Booster lattice. The corresponding values of the incoming (horizontal) beam ellipse parameters are

$$
\alpha_{0}=-0.5208, \quad \beta_{0}=3.288 \text { meters. }
$$

In [1] these parameter values were shown to give favorable results for the injection of beam ellipses with emittance $11 \pi \mathrm{mm}$ milliradians.

Figure 11 shows horizontal beam envelopes in the inflector channel for the case $A=0$ and $B=0.3$. The blue, red, brown, and violet curves show the envelopes for incoming beam ellipse emittances $11 \pi, 22 \pi, 33 \pi$, and $44 \pi$ $\mathrm{mm}$ milliradians respectively. The $22 \pi$ beam ellipse just fits through the 
channel if the half-aperture is $8.5 \mathrm{~mm}$. The $33 \pi$ beam ellipse fits if the half-aperture is increased to $10.5 \mathrm{~mm}$.

Figures 12 through 14 show the beam and machine ellipses for the case $A=0$ and $B=0.3$.

In Figure 12 the beam ellipse has emittance $11 \pi \mathrm{mm}$ milliradians. The beam ellipse ends up safely inside the QHC4 quadrupole aperture. The solid and dashed brown ellipses are well inside the black machine ellipse border.

In Figure 13 the beam ellipse emittance has been increased to $22 \pi \mathrm{mm}$ milliradians. The red beam ellipse is again safely inside the QHC4 quadrupole aperture. The solid and dashed brown ellipses are again well inside the black machine ellipse border. The black dashed ellipse is matched to the lattice at the inflector exit and has emittance $\pi \epsilon_{M}=\pi A_{M}^{2} / \beta_{M}$ where $A_{M}=76 \mathrm{~mm}$ is the half-aperture and $\beta_{M}=14 \mathrm{~m}$ is the value of the beta function in the Booster quadrupoles. This shows that the incoming beam ellipse would stay inside the quadrupole apertures if it were able to circulate around the machine with the injection dipoles turned off.

In Figure 14 the beam ellipse emittance has been increased to $33 \pi \mathrm{mm}$ milliradians. The inflector half-aperture also has been increased from 8.5 to $10.5 \mathrm{~mm}$ to accommodate the larger beam ellipse. The inflector septum (outer side) then moves in from $X=48.5$ to $46.5 \mathrm{~mm}$. The red beam ellipse is now close to but still inside the QHC4 quadrupole aperture. The solid and dashed brown ellipses are getting closer to the black machine ellipse border, showing that the injection bump needs to be collapsed more quickly away from the septum in order to keep the beam ellipse from hitting the septum. Some parts of the black beam ellipse are now outside the black dashed ellipse. These parts would be lost on quadrupole apertures if the beam ellipse were able to circulate around the machine with the injection dipoles turned off.

In Figure 15 the beam ellipse emittance has been increased further to $44 \pi$ mm milliradians. The parameter $B$ has been reduced from 0.3 to 0.26537 so that the beam ellipse will clear the QHC4 aperture. The inflector half-aperture has been increased to $12 \mathrm{~mm}$ to accommodate the larger beam ellipse. The inflector septum (outer side) then moves in from $X=48.5$ to $45.0 \mathrm{~mm}$. The red beam ellipse is close to but still inside the QHC4 quadrupole aperture. The solid and dashed brown ellipses are now very close to the black machine ellipse border, showing that the injection 
bump needs to be collapsed even more quickly away from the septum in order to keep the beam ellipse from hitting the septum. A larger portion of the black beam ellipse is outside the black dashed ellipse. These parts would be lost on quadrupole apertures if the beam ellipse were able to circulate around the machine with the injection dipoles turned off.

\section{Conditions for Injection and Storage of Large Emittance Beams without Loss}

Let us now find conditions for which beam ellipses with parameters (46) and emittances from $22 \pi$ to $33 \pi \mathrm{mm}$ milliradians can be injected and stored in Booster without loss. Following the analysis of [1] we obtain the results shown in Figures $\mathbf{1 6}$ through $\mathbf{3 1}$. Here the beam ellipse is launched from the inflector exit as the injection bump is collapsed linearly over a small number of turns. A one-turn beam pulse from EBIS is assumed. The turn-by-turn evolution of the beam ellipse is calculated for launches at the beginning and end of the beam pulse. The magnetic rigidity is taken to be 1.255 Tm which is the expected maximum for EBIS beams.

Figures 16 through 23 show the setup for injecting and storing a $22 \pi \mathrm{mm}$ milliradian beam ellipse without loss on the inflector septum. The maximum rate of fall of current required in the injection dipoles is 9.651 $\mathrm{A} / \mu \mathrm{s}$. This is beyond the practical limit of $6 \mathrm{~A} / \mu \mathrm{s}$ but within the advertised limit of $12 \mathrm{~A} / \mu \mathrm{s}$ [1]. (The practical limit has been set to protect power supply transistors.) As shown by Figures 20 and 23, the beam ellipses launched at the beginning and end of the beam pulse end up inside the Booster acceptance after the injection bump has collapsed. The required Booster acceptance can be achieved by moving the closed orbit away from the beam dump [6] in the B6 straight, away from the H-minus injection foil in the C6 straight, and away from the extraction septum magnet in the F6 straight.

Figures 24 through 31 show the setup for injecting and storing a $33 \pi \mathrm{mm}$ milliradian beam ellipse without loss on the inflector septum. Here the maximum rate of fall of current required in the injection dipoles is 12 $\mathrm{A} / \mu \mathrm{s}$. The closed orbit has been offset so that it is $7 \mathrm{~mm}$ to the inside of the beam pipe centerline when the current in the injection dipoles is zero. This is needed to keep the beam ellipse launched at the end of the beam pulse away from the septum. Figures $\mathbf{2 8}$ and $\mathbf{3 1}$ show that the beam ellipses launched at the beginning and end of the beam pulse end up inside 
the Booster acceptance after the injection bump has collapsed.

\section{$7 \quad$ Inflector Voltage}

The inflector voltage $V_{I}$ required for the injection of particles of mass $m$, charge $e Q$, and velocity $c \beta$ is

$$
e V_{I}=\frac{G}{R_{I}}\left(\frac{m c^{2}}{Q}\right) \beta^{2} \gamma
$$

where $e$ is the elementary charge and

$$
\gamma=1 / \sqrt{1-\beta^{2}}
$$

The present gap between the cathode and septum of the inflector is

$$
G=2 a=0.017 \text { meters }
$$

and the radius of curvature along the nominal trajectory is

$$
R_{I}=\rho=8.74123 \text { meters. }
$$

For ions from EBIS we have

$$
\beta^{2} \gamma \leq 0.00429948
$$

and

$$
\frac{m c^{2}}{Q} \leq 6 \mathrm{GeV}
$$

The maximum required voltage is then

$$
V_{I}=50.1699 \mathrm{kV} \text {. }
$$

If the gap $G$ is increased from 17 to $21 \mathrm{~mm}$, the maximum required voltage becomes

$$
V_{I}=61.9746 \mathrm{kV} .
$$

This is comfortably within the operating range of the present power supply. (For the deuteron-gold run in 2008 the supply was run at $72 \mathrm{kV}$ without any problems.) 


\section{Summary of Desired Beam Ellipse Parameters at Inflector Exit}

\subsection{Horizontal Parameters}

The horizontal Courant-Snyder parameters of the Booster lattice at the inflector exit are

$$
\alpha=-1.736, \quad \beta=10.96 \text { meters. }
$$

In the previous sections we have shown that it is desirable for the incoming beam ellipse to have horizontal Courant-Snyder parameters

$$
\alpha_{0}=B \alpha, \quad \beta_{0}=B \beta
$$

at the inflector exit, where

$$
B=0.3 \text {. }
$$

This gives

$$
\alpha_{0}=-0.5208, \quad \beta_{0}=3.288 \text { meters } .
$$

\subsection{Vertical Parameters}

In [2] we showed that it is desirable for the incoming beam ellipse to have vertical Courant-Snyder parameters matched to those of the Booster lattice at the inflector exit. These are

$$
\alpha_{V}=0.823, \quad \beta_{V}=4.761 \text { meters. }
$$

\subsection{Dispersion Parameters}

In [2] we showed that it is desirable for the dispersion $d$ and its derivative $d^{\prime}$ to be zero at the inflector exit. 


\section{References}

[1] C.J. Gardner, "Multiturn Injection of EBIS Ions in Booster", C-A/AP/Note 405, September 2010.

[2] C.J. Gardner, "Notes on the Injection of EBIS Ions into Booster", C-A/AP/Note 240, June 2006.

[3] C.J. Gardner, "Booster Inflector Specifications", Booster Tech. Note 159, February 28, 1990

[4] C. Bovet, et al, "A Selection of Formulae and Data Useful for the Design of A.G. Synchrotrons", CERN/MPS-SI/Int. DL/70/4, 23 April, 1970 .

[5] D. A. Edwards and M. J. Syphers, "An Introduction to the Physics of High Energy Accelerators", Wiley \& Sons, New York, 1993

[6] C.J. Gardner, "The New Booster Dump and Dump Bumps", C-A/AP/Note 46, March 2001. 


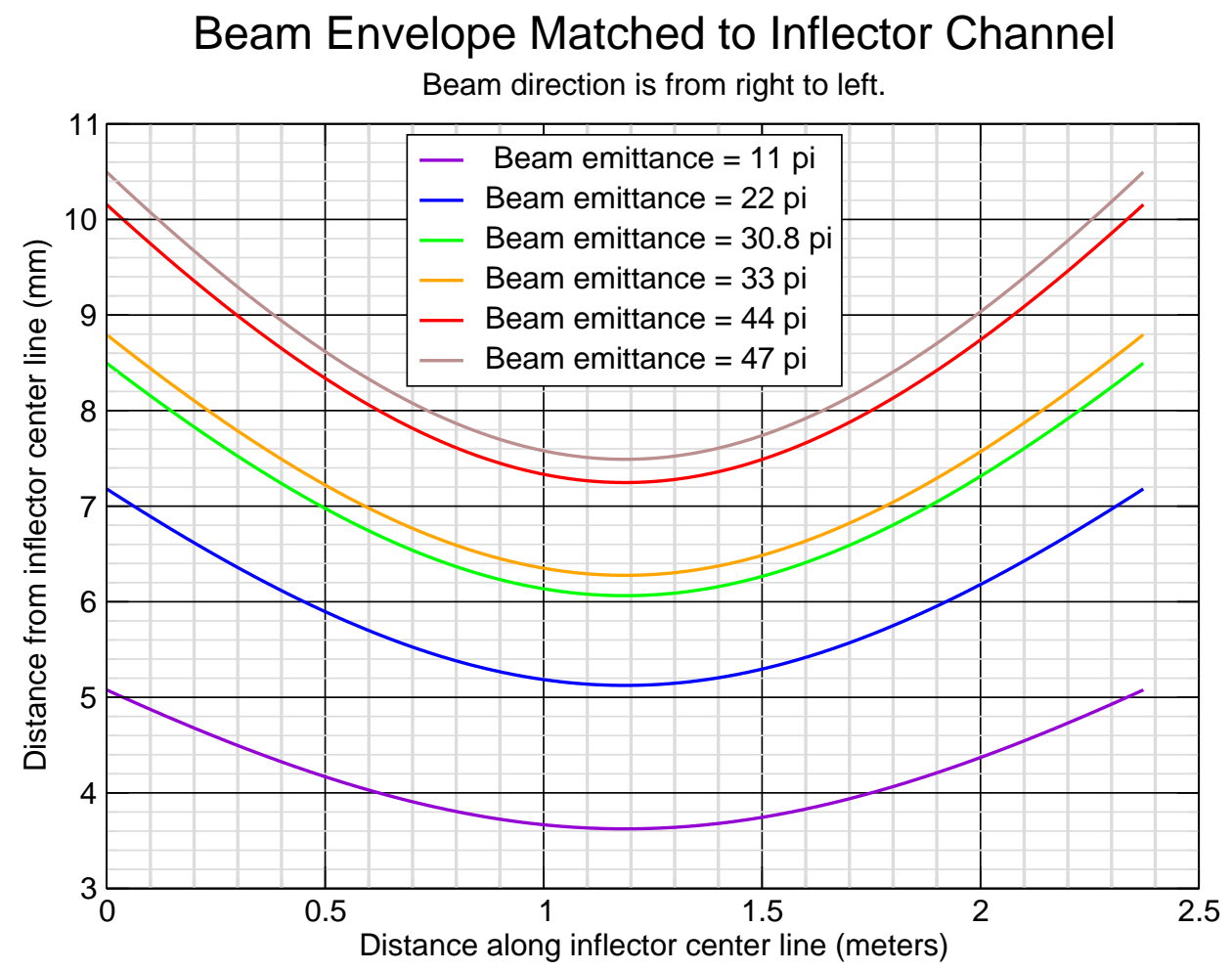

Figure 1: Horizontal beam envelope $\sqrt{\epsilon_{0} \beta}$ in the inflector channel for the case in which the beam ellipse is matched to the inflector channel. Beam direction is from right to left with the inflector exit on the left. The violet, blue, orange, and red curves show the envelope for beam ellipse emittances $\pi \epsilon_{0}=11 \pi, 22 \pi, 33 \pi$, and $44 \pi \mathrm{mm}$ milliradians respectively. The green and brown curves show the envelope for $\pi \epsilon_{0}=30.8 \pi$ and $47.0 \pi \mathrm{mm}$ milliradians respectively. The horizontal half-aperture of the existing channel is $8.5 \mathrm{~mm}$. This gives a maximum acceptance of $\mathbf{3 0 . 8}$ pi $\mathbf{~ m m ~ m i l l i r a d i a n s . ~ I f ~ t h e ~ h a l f - a p e r t u r e ~ i s ~ i n c r e a s e d ~ t o ~} 10.5 \mathrm{~mm}$, the maximum acceptance becomes 47.0 pi mm milliradians. 


\section{Beam and Machine Ellipses}

Incoming beam ellipse emittance is 11 pi matched to inflector channel

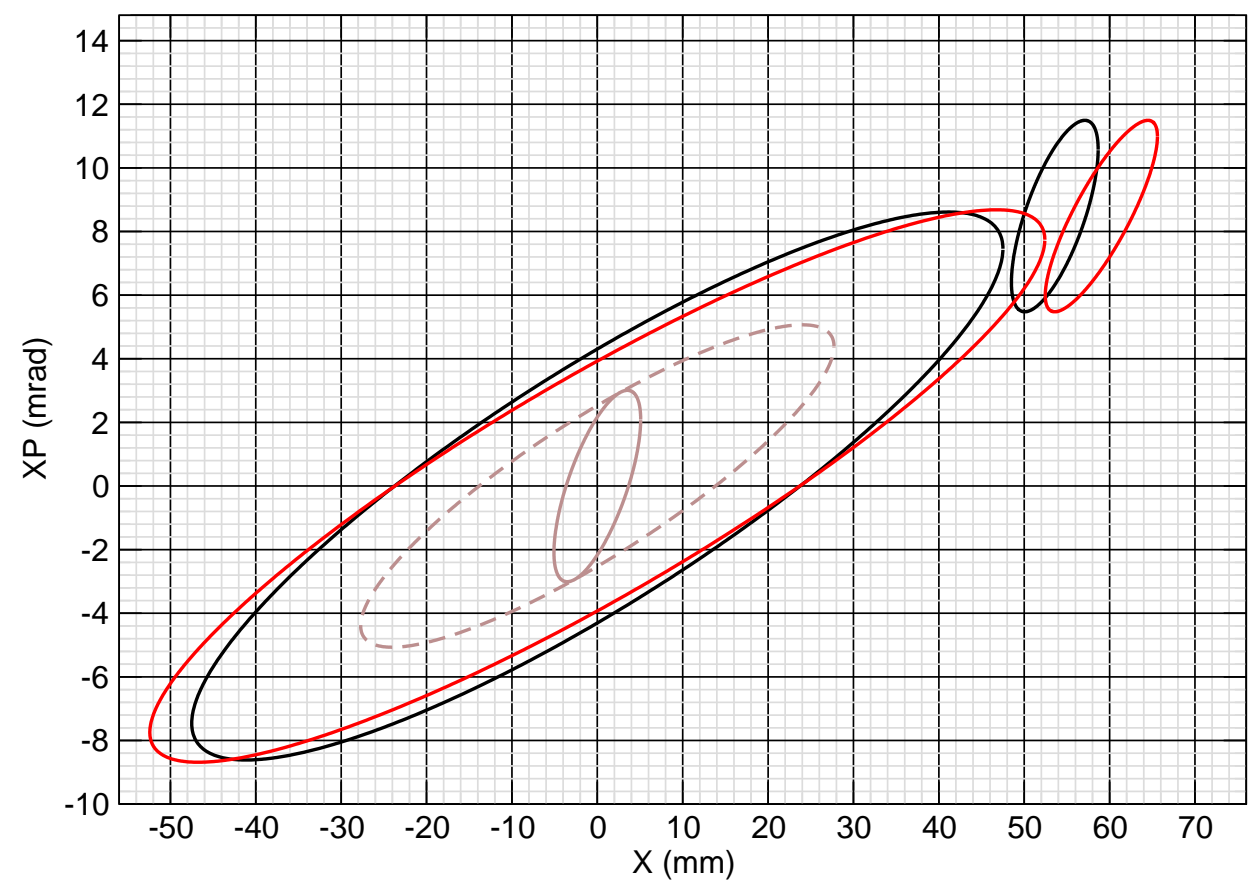

Figure 2: Here the large and small black ellipses are the machine and beam ellipses, respectively, at the inflector exit. These evolve into the red ellipses in the 0.64253 meter drift from the inflector exit to quadrupole QHC4 of the Booster lattice. The half-aperture of the quadrupole is $76 \mathrm{~mm}$. The beam ellipse has emittance $\pi \epsilon_{0}=11 \pi \mathrm{mm}$ milliradians and is matched to the inflector channel. It ends up safely inside the QHC4 quadrupole aperture. The black machine ellipse is centered on the Booster beam pipe and just touches the septum of the inflector. The outer side of the $1 \mathrm{~mm}$ thick septum is $48.5 \mathrm{~mm}$ from the center of the beam pipe. The solid brown ellipse is the incoming beam ellipse shifted to the center of the machine ellipse. The dashed brown ellipse is the smallest machine ellipse that contains the beam ellipse. Its emittance, $\pi \epsilon$, is given by the mismatch factor $\epsilon / \epsilon_{0}=6.37$. 


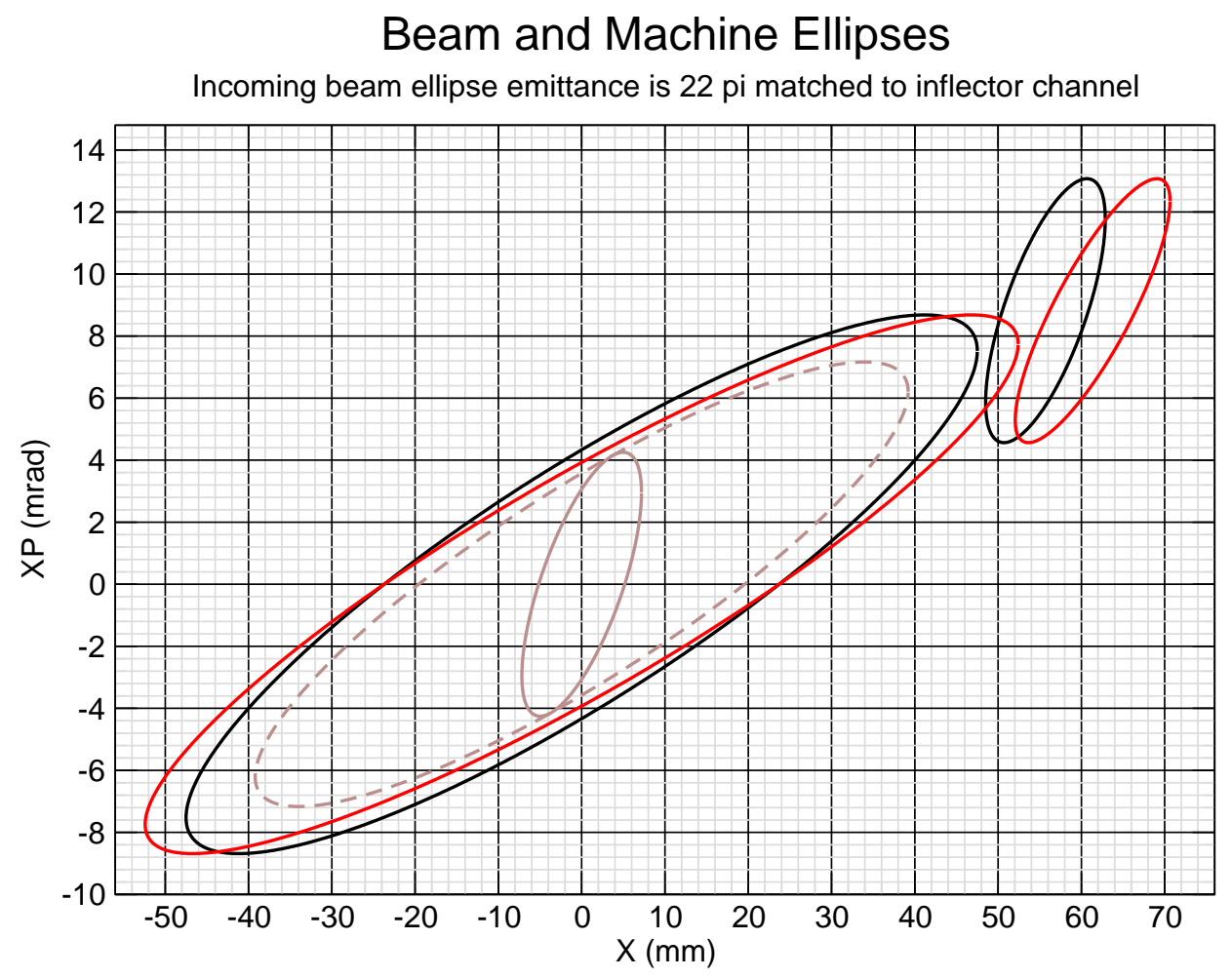

Figure 3: Here the incoming beam ellipse emittance has been increased to $\pi \epsilon_{0}=22 \pi \mathrm{mm}$ milliradians. This ellipse again ends up safely inside the QHC4 quadrupole aperture at $76 \mathrm{~mm}$. The solid and dashed brown ellipses are still inside but getting close to the black machine ellipse border. 


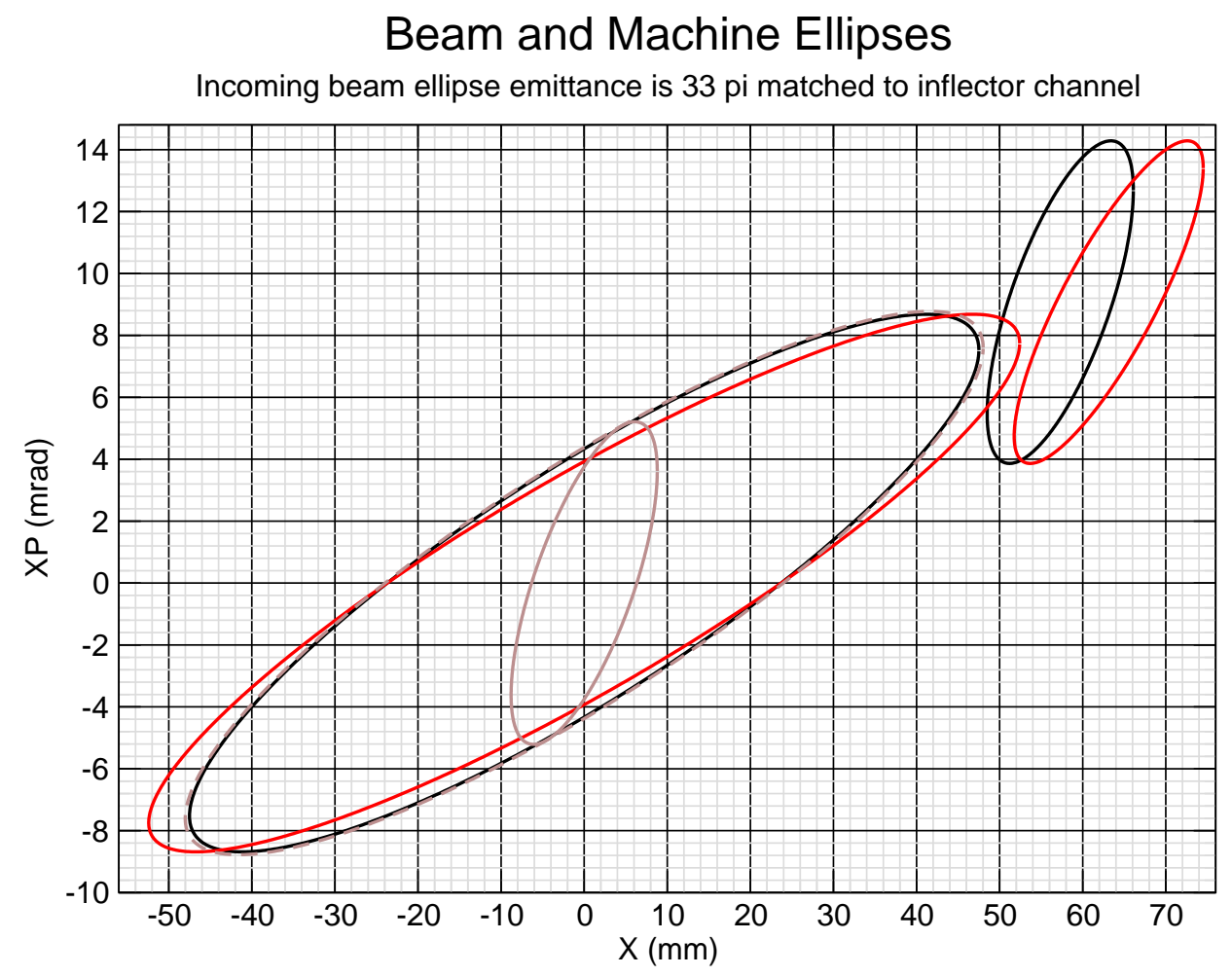

Figure 4: Here the incoming beam ellipse emittance has been increased to $\pi \epsilon_{0}=33 \pi \mathrm{mm}$ milliradians. This ellipse ends up close to the QHC4 quadrupole aperture. The solid and dashed brown ellipses now extend a little beyond the black machine ellipse border. 


\section{Beam Envelope in Inflector Matched to Machine Lattice}

Beam direction is from right to left.

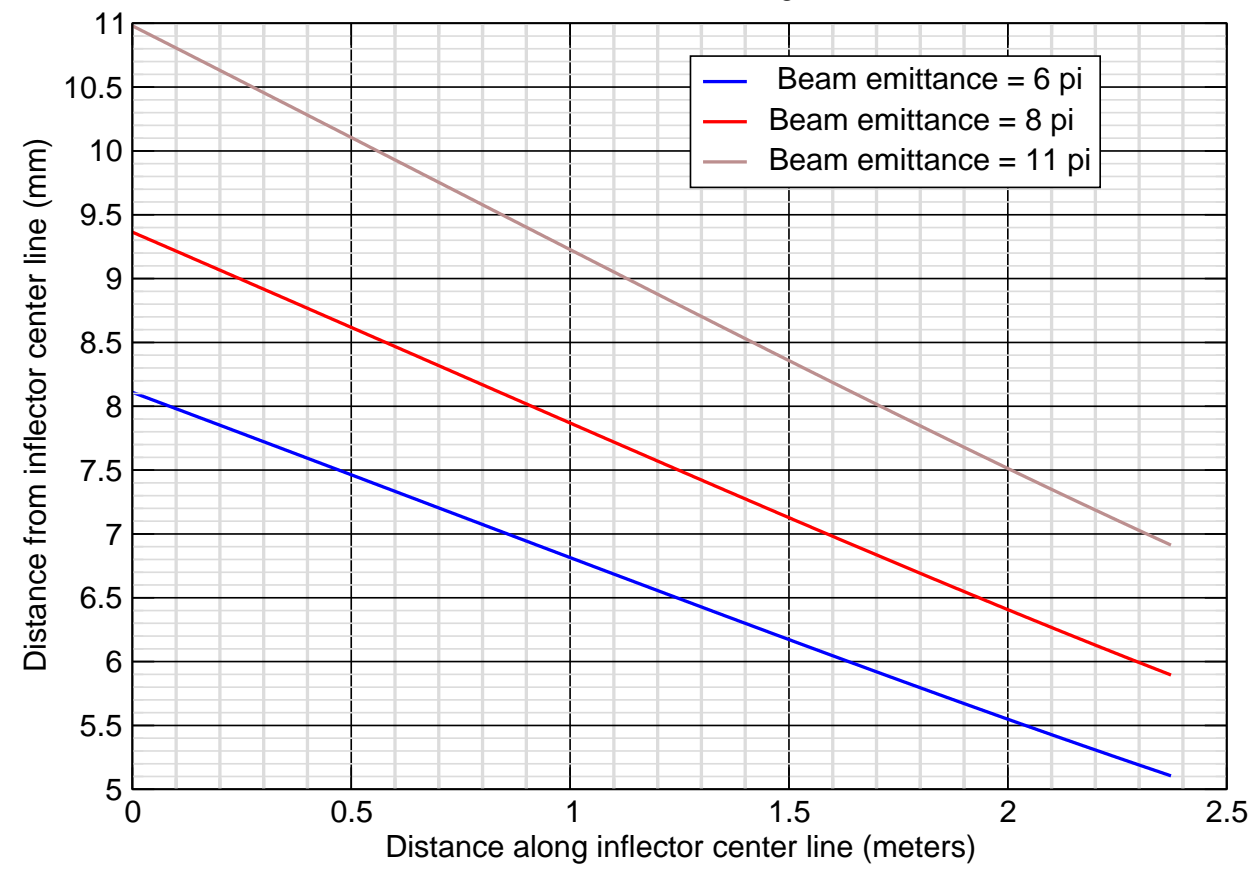

Figure 5: Horizontal beam envelope in the inflector channel for the case in which the beam ellipse is matched to the machine lattice. Beam direction is from right to left with the inflector exit on the left. The blue, red and brown curves show the envelope for beam ellipse emittances $6 \pi, 8 \pi$ and $11 \pi \mathrm{mm}$ milliradians respectively. The horizontal half-aperture of the existing channel is $8.5 \mathbf{~ m m}$. This gives a maximum acceptance of only $6.59 \mathrm{pi} \mathrm{mm}$ milliradians. If the half-aperture is increased to 10.5 $\mathrm{mm}$, the acceptance increases to $10.1 \mathrm{pi} \mathrm{mm}$ milliradians. 


\section{Beam and Machine Ellipses}

Incoming beam ellipse emittance is 6 pi matched to machine lattice

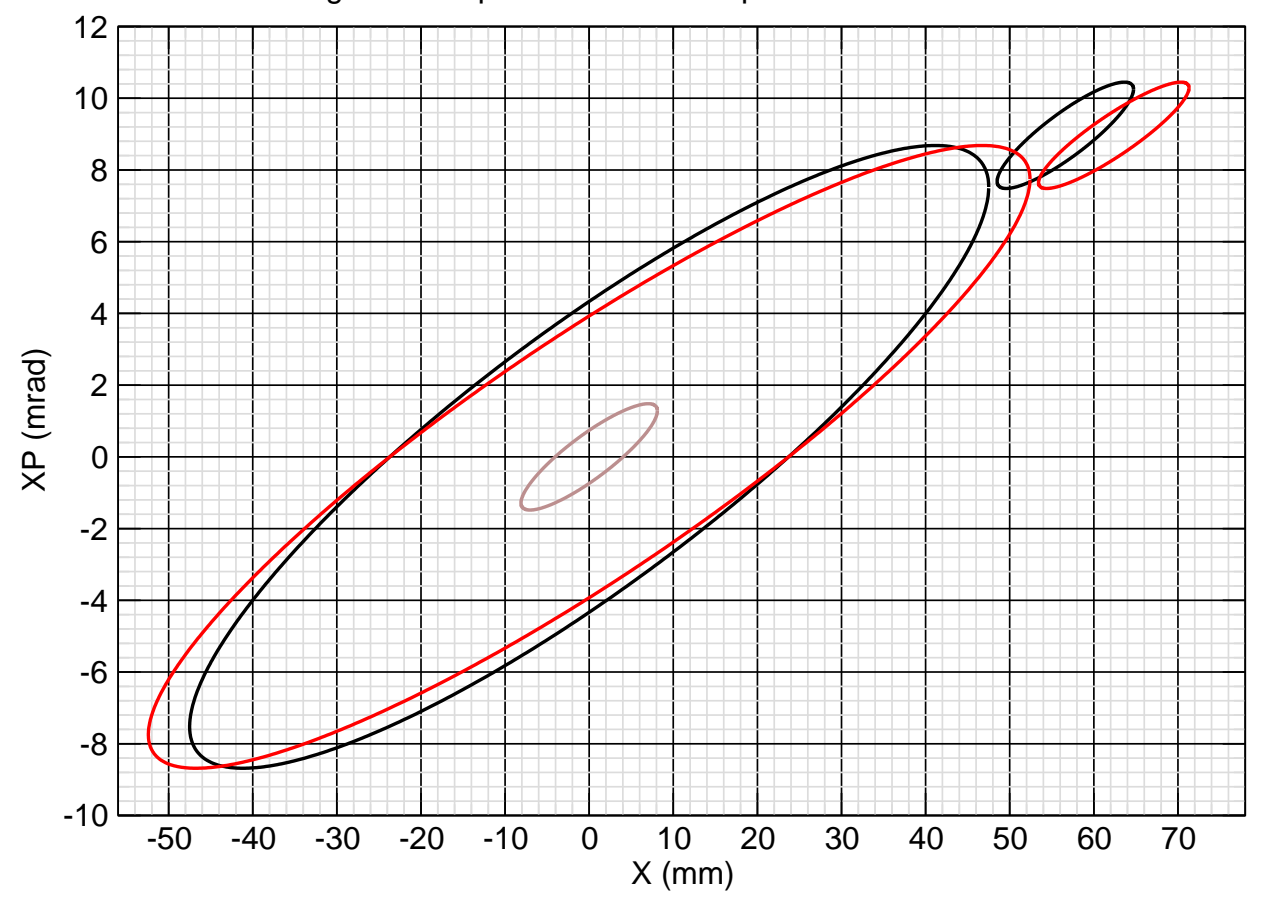

Figure 6: Here the black ellipses are again the machine and incoming beam ellipses at the inflector exit. These evolve into the red ellipses in the drift to the QHC4 quadrupole. The half-aperture of the quadrupole is $76 \mathrm{~mm}$. The beam ellipse has emittance $\pi \epsilon_{0}=6 \pi \mathrm{mm}$ milliradians and is matched to the machine lattice. It ends up safely inside the QHC4 quadrupole aperture. The brown ellipse is the incoming beam ellipse shifted to the center of the black machine ellipse. 


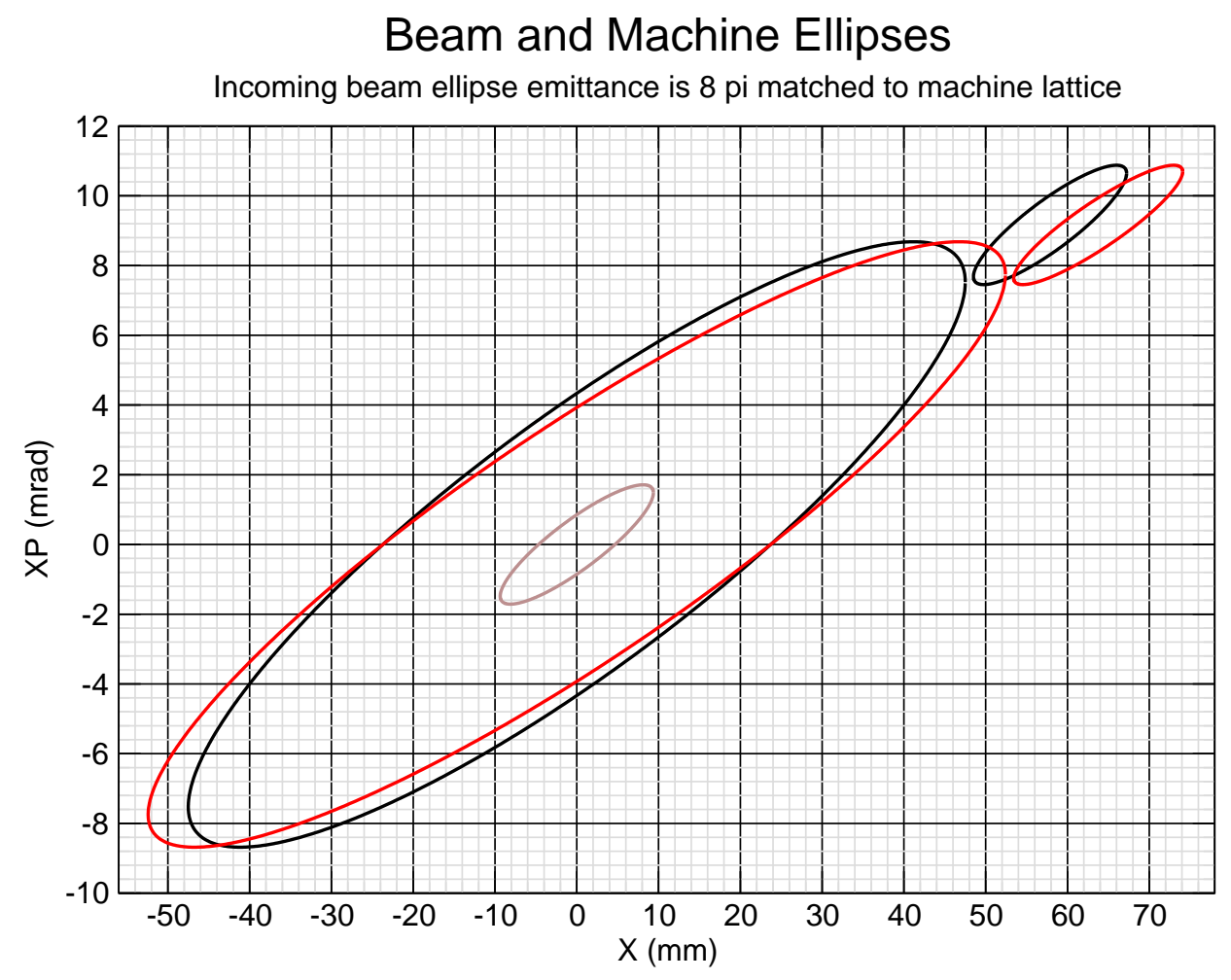

Figure 7: Here the beam ellipse emittance has been increased to $8 \pi \mathrm{mm}$ milliradians. This ellipse ends up safely inside but close to the QHC4 quadrupole aperture. 


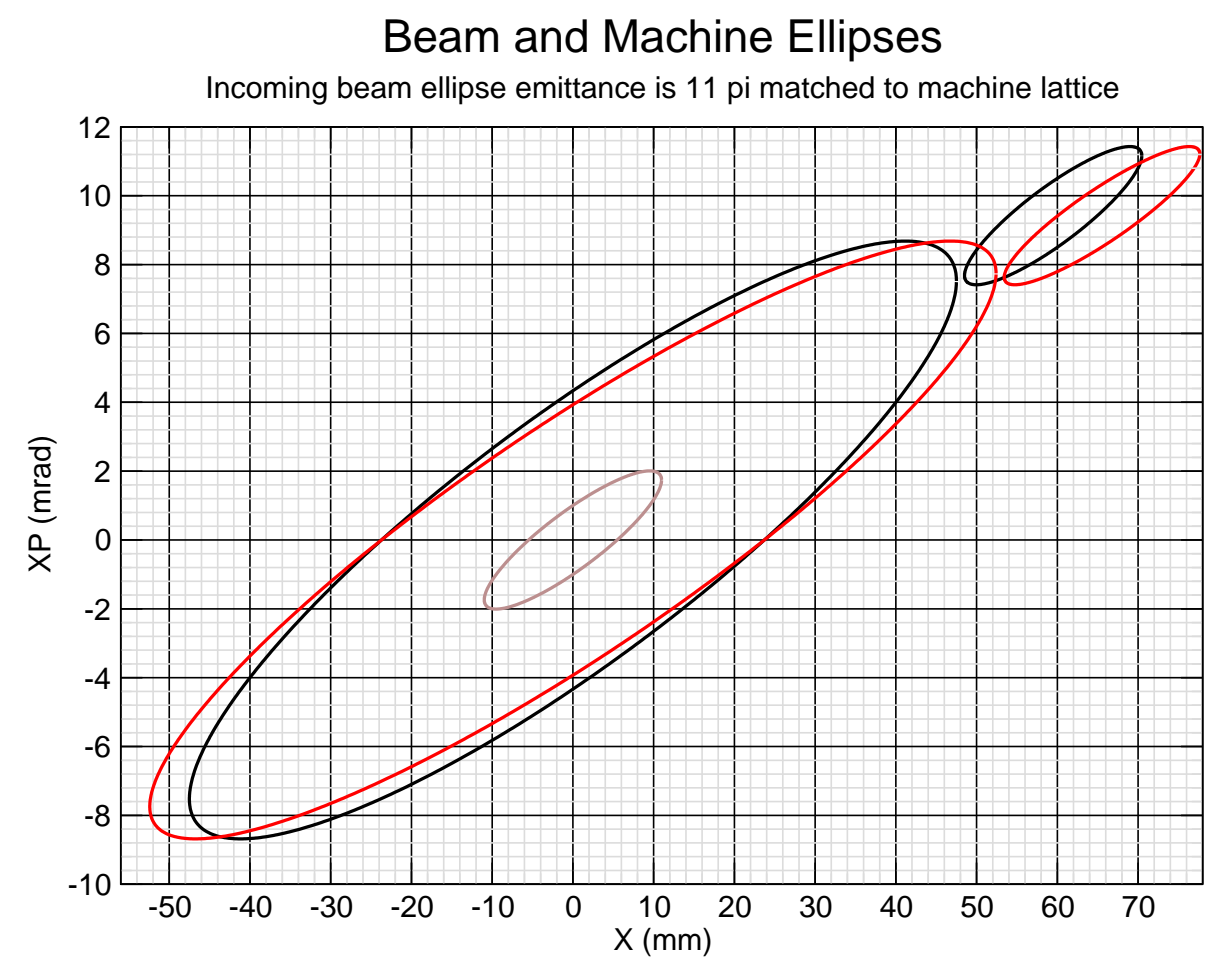

Figure 8: Here the beam ellipse emittance has been increased to $11 \pi \mathrm{mm}$ milliradians. This ellipse ends up scraping against the QHC4 quadrupole aperture. 


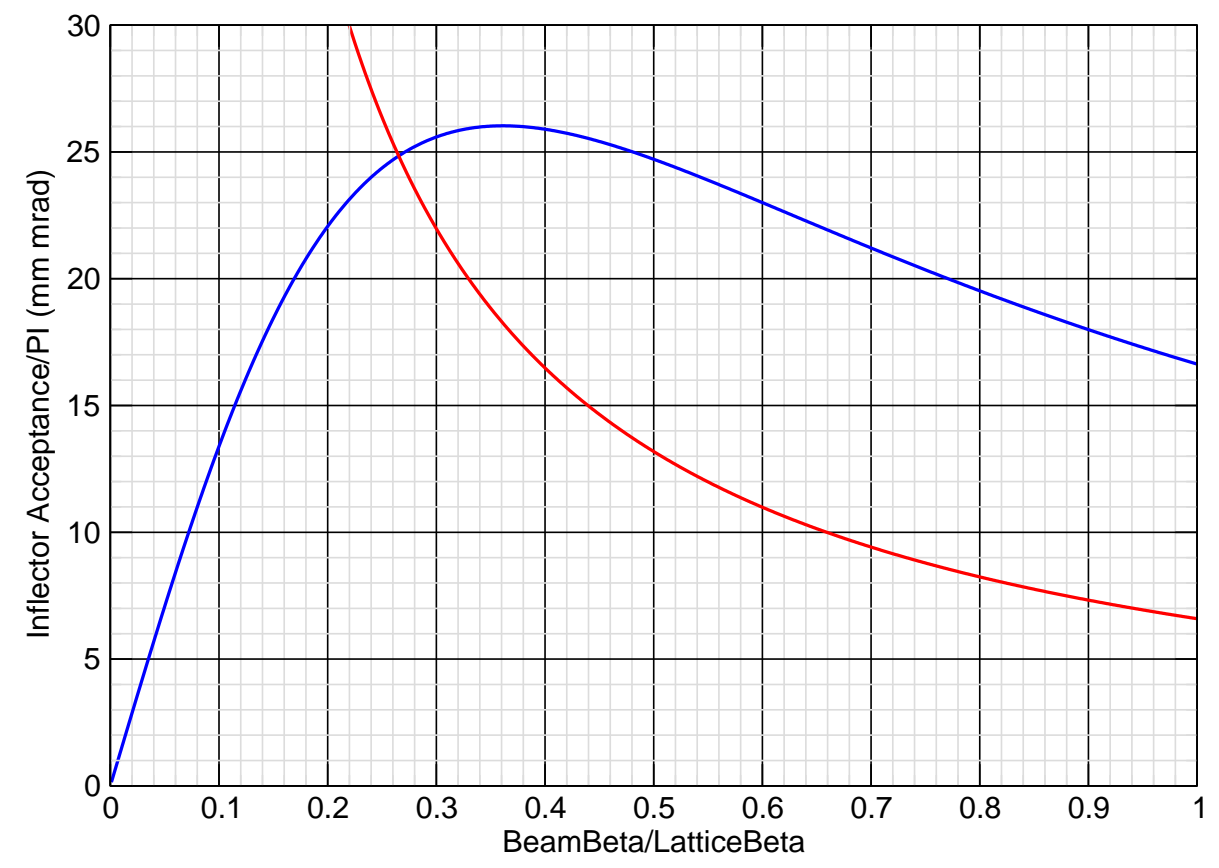

Figure 9: Inflector horizontal acceptance as a function of the parameter $B=$ $\beta_{0} / \beta$. Here we have taken $A=0$, which minimizes the mismatch factor for any given $B$. The red curve is $e_{0}=a^{2} / \beta_{0}$ and the blue curve is $e_{1}=a^{2} / \beta_{1}$. The half-aperture is taken to be $a=8.5 \mathrm{~mm}$. The two curves intersect at $B=0.26537$ which gives the maximum acceptance $\pi e_{0}=\pi e_{1}=24.8 \pi \mathrm{mm}$ milliradians. For $B \leq 0.26537$, the acceptance is given by the blue curve. For $B \geq 0.26537$, the acceptance is given by the red curve. 


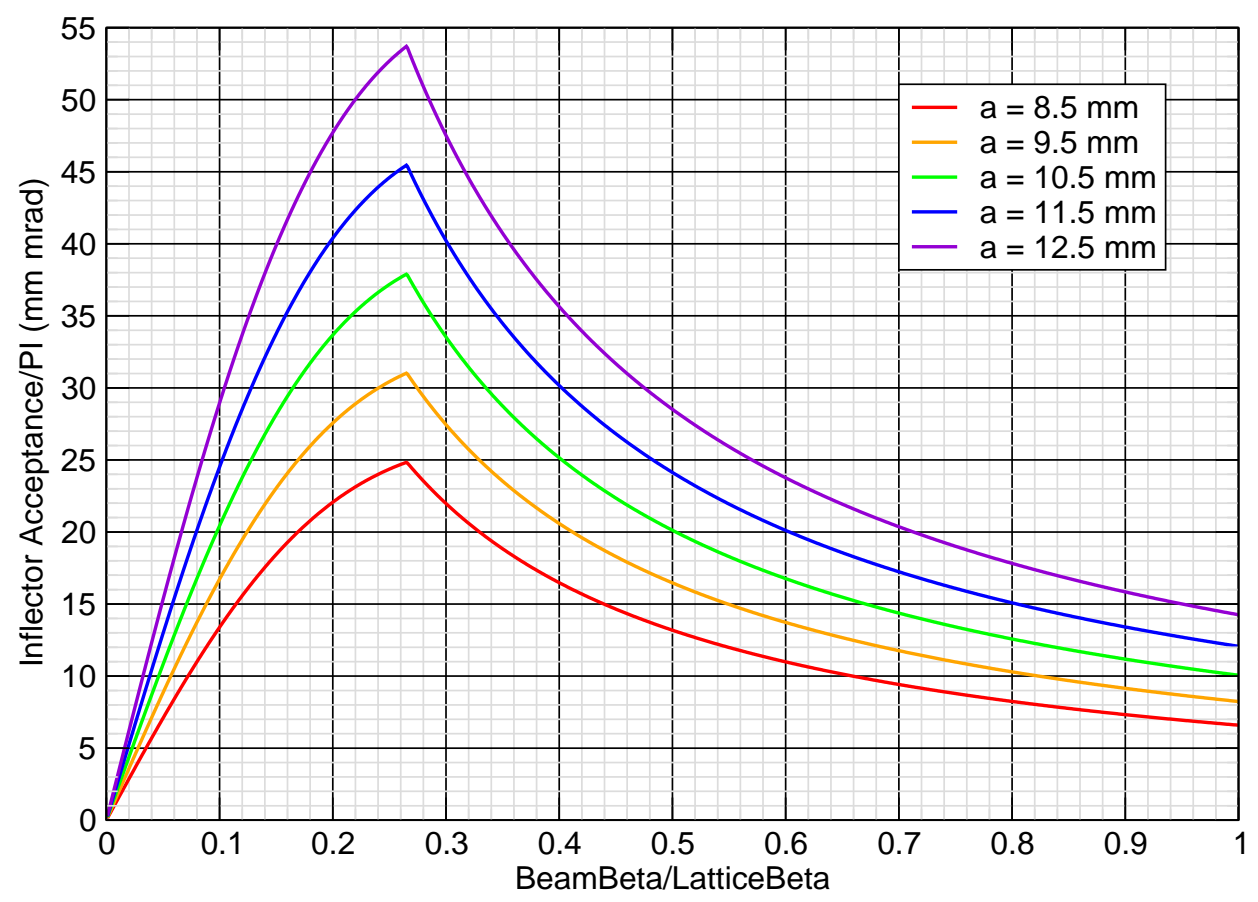

Figure 10: Inflector horizontal acceptance as a function of the parameter $B=\beta_{0} / \beta$ for various values of the horizontal half-aperture $a$. As before we have taken $A=0$, which minimizes the mismatch factor for any given $B$. The red, orange, green, blue, and violet curves give the acceptance for horizontal half-apertures $a=8.5,9.5,10.5,11.5$ and $12.5 \mathrm{~mm}$ respectively. The horizontal half-aperture of the existing channel is $8.5 \mathrm{~mm}$. For each curve the maximum acceptance occurs at $B=0.26537$. This value of $B$ gives mismatch factor $\epsilon / \epsilon_{0}=3.77$. For $a=8.5 \mathrm{~mm}$, the maximum acceptance is 24.8 pi $\mathbf{~ m m ~ m i l l i r a d i a n s . ~ F o r ~} a=10.5 \mathrm{~mm}$, the maximum is $\mathbf{3 7 . 9} \mathbf{~ p i}$ mm milliradians. For $B=0.3$, the red curve shows that the acceptance is 22.0 pi $\mathbf{~ m m ~ m i l l i r a d i a n s ~ f o r ~ h a l f - a p e r t u r e ~} a=8.5 \mathrm{~mm}$. If the halfaperture is increased to $10.5 \mathrm{~mm}$, the acceptance increases to $33.5 \mathbf{p i ~} \mathbf{~ m m}$ milliradians as shown by the green curve. 


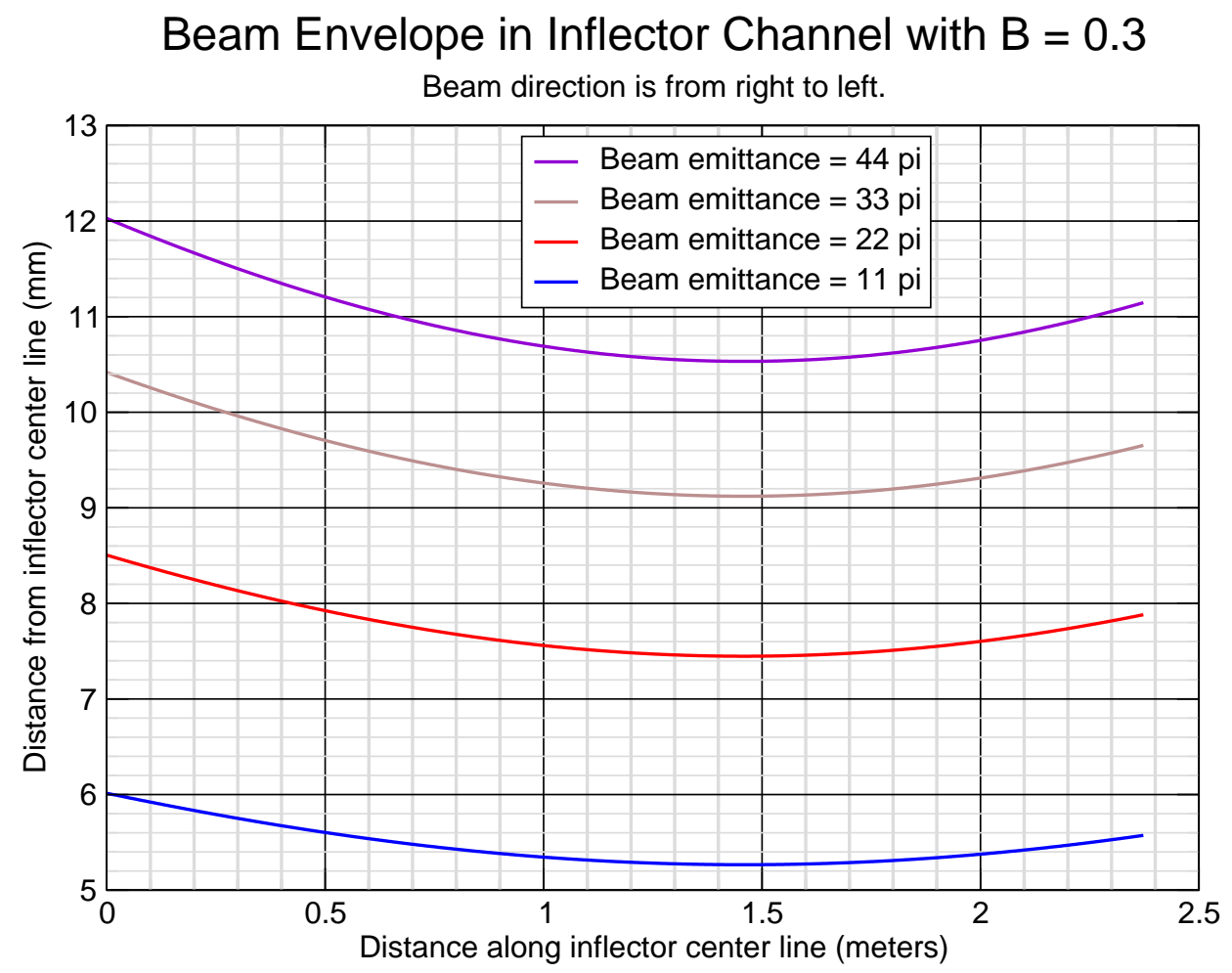

Figure 11: Horizontal beam envelopes in the inflector channel for the case in which $A=0$ and $B=0.3$. Beam direction is again from right to left with the inflector exit on the left. The blue, red, brown, and violet curves show the envelopes for incoming beam emittances $11 \pi, 22 \pi, 33 \pi$, and $44 \pi$ $\mathrm{mm}$ milliradians respectively. The horizontal half-aperture of the existing channel is $8.5 \mathrm{~mm}$. This gives an acceptance of $\mathbf{2 2 . 0} \mathbf{~ p i ~} \mathbf{~ m m}$ milliradians. If the half-aperture is increased to $10.5 \mathrm{~mm}$, the acceptance increases to 33.5 pi mm milliradians. 


\section{Beam and Machine Ellipses}

Incoming beam ellipse emittance is 11 pi with $B=0.3$

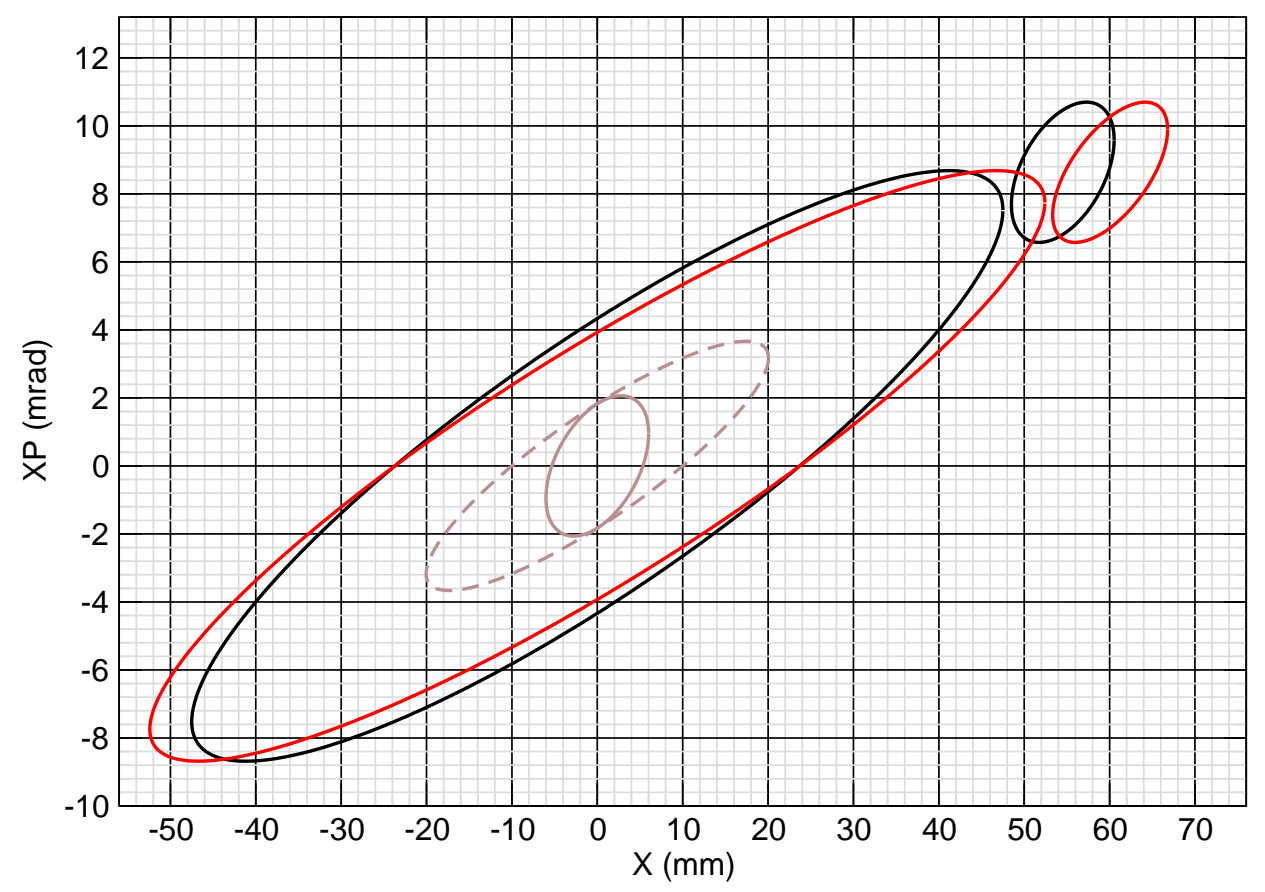

Figure 12: Here, as before, the large and small black ellipses are the machine and beam ellipses, respectively, at the inflector exit. These evolve into the red ellipses in the 0.64253 meter drift from the inflector exit to quadrupole QHC4 of the Booster lattice. The half-aperture of the quadrupole is 76 $\mathrm{mm}$. The incoming beam ellipse has parameters $A=0$ and $B=0.3$ and emittance $\pi \epsilon_{0}=11 \pi \mathrm{mm}$ milliradians. It ends up safely inside the QHC4 quadrupole aperture. The black machine ellipse is centered on the Booster beam pipe and just touches the septum of the inflector. The outer side of the $1 \mathrm{~mm}$ thick septum is $48.5 \mathrm{~mm}$ from the center of the beam pipe. The solid brown ellipse is the incoming beam ellipse shifted to the center of the machine ellipse. The dashed brown ellipse is the smallest machine ellipse that contains the beam ellipse. It is well inside the black machine ellipse. 


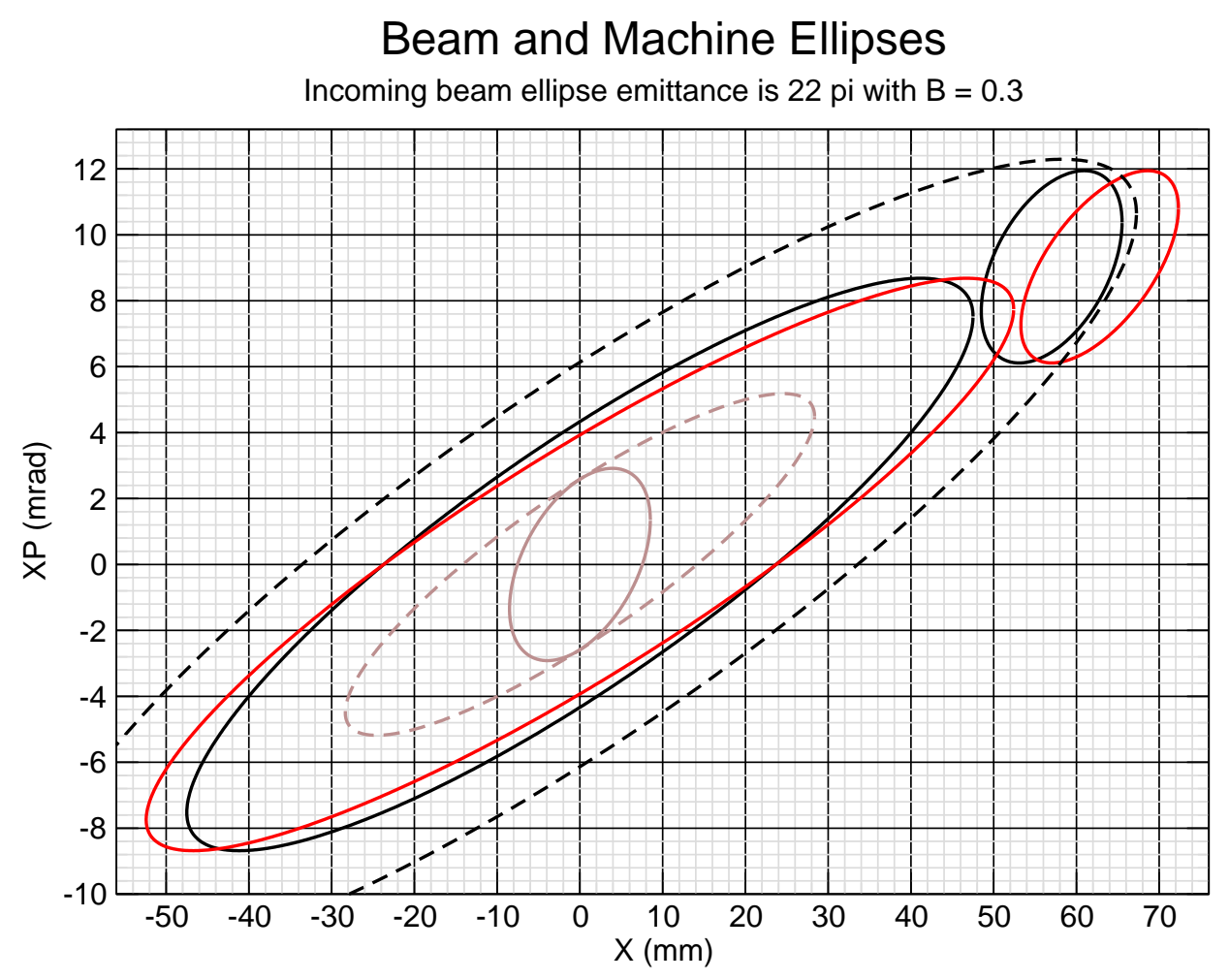

Figure 13: Same as Figure 12 but with incoming beam ellipse emittance $\pi \epsilon_{0}=22 \pi \mathrm{mm}$ milliradians. This ellipse again ends up safely inside the QHC4 quadrupole aperture. The brown dashed ellipse is again well inside the black machine ellipse. The black dashed ellipse is matched to the lattice at the inflector exit and has emittance $\pi \epsilon_{M}=\pi A_{M}^{2} / \beta_{M}$ where $A_{M}=76$ $\mathrm{mm}$ is the half-aperture and $\beta_{M}=14 \mathrm{~m}$ is the value of the beta function in the Booster quadrupoles. This shows that the incoming beam ellipse would stay inside the quadrupole apertures if it were able to circulate around the machine with the injection dipoles turned off. 


\section{Beam and Machine Ellipses}

Incoming beam ellipse emittance is 33 pi with $B=0.3$

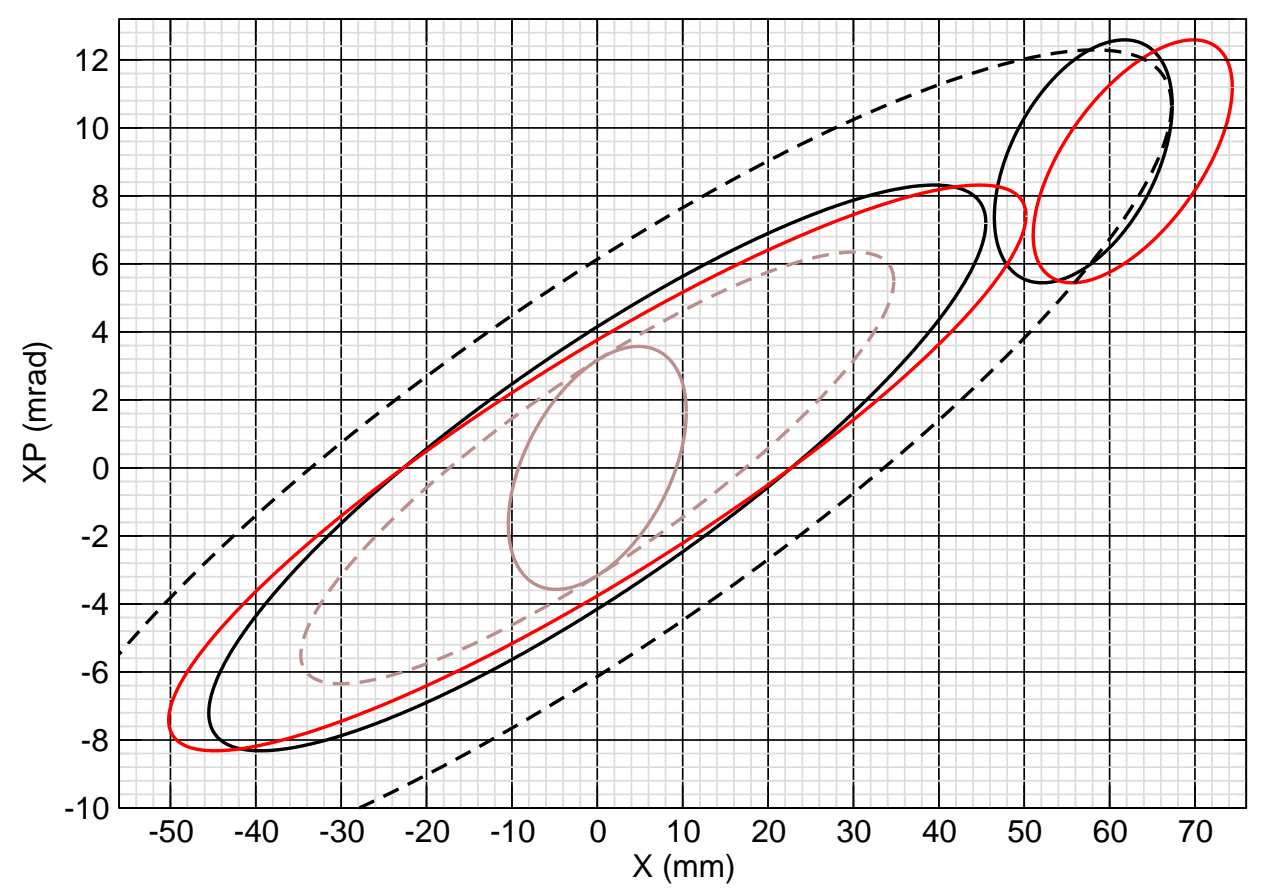

Figure 14: Same as Figure 12 but with incoming beam ellipse emittance $\pi \epsilon_{0}=33 \pi \mathrm{mm}$ milliradians. The inflector half-aperture has been increased from 8.5 to $10.5 \mathrm{~mm}$ to accommodate the larger beam ellipse. The inflector septum (outer side) then moves from $X=48.5$ to $46.5 \mathrm{~mm}$. The red beam ellipse is now close to but still inside the QHC4 quadrupole aperture. The solid and dashed brown ellipses are getting closer to the black machine ellipse border, showing that the injection bump needs to be collapsed more quickly away from the septum in order to keep the beam ellipse from hitting the septum. Some parts of the black beam ellipse are now outside the black dashed ellipse. These parts would be lost on quadrupole apertures if the beam ellipse were able to circulate around the machine with the injection dipoles turned off. 


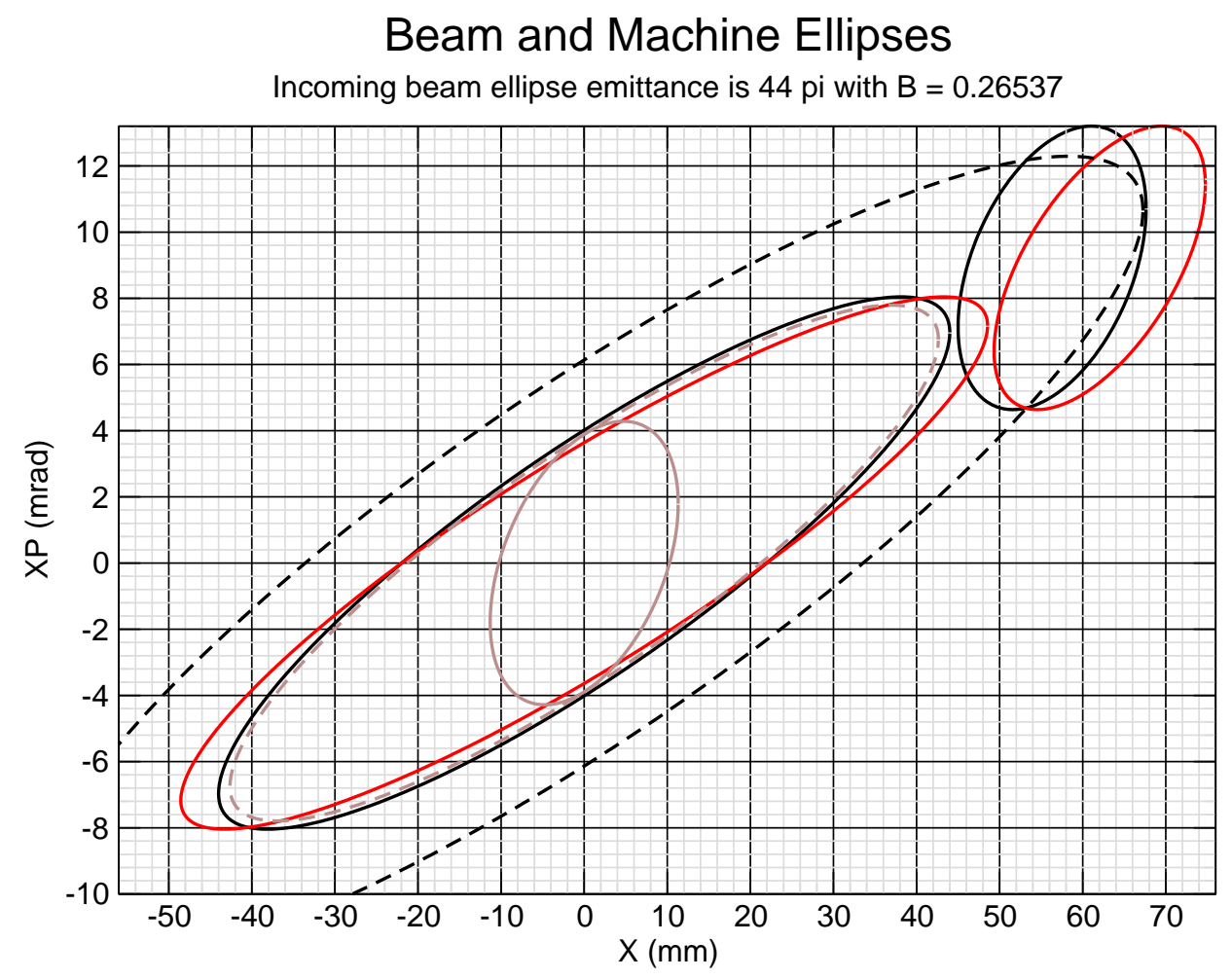

Figure 15: Here the beam ellipse emittance has been increased further to $\pi \epsilon_{0}=44 \pi \mathrm{mm}$ milliradians. The parameter $B$ has been reduced from 0.3 to 0.26537 so that the beam ellipse will clear the QHC4 aperture. The inflector half-aperture has been increased to $12 \mathrm{~mm}$ to accommodate the larger beam ellipse. The inflector septum (outer side) then moves in from $X=48.5$ to $45.0 \mathrm{~mm}$. The red beam ellipse is close to but still inside the QHC4 quadrupole aperture. The solid and dashed brown ellipses are now very close to the black machine ellipse border, showing that the injection bump needs to be collapsed even more quickly away from the septum in order to keep the beam ellipse from hitting the septum. A larger portion of the black beam ellipse is outside the black dashed ellipse. These parts would be lost on quadrupole apertures if the beam ellipse were able to circulate around the machine with the injection dipoles turned off. 


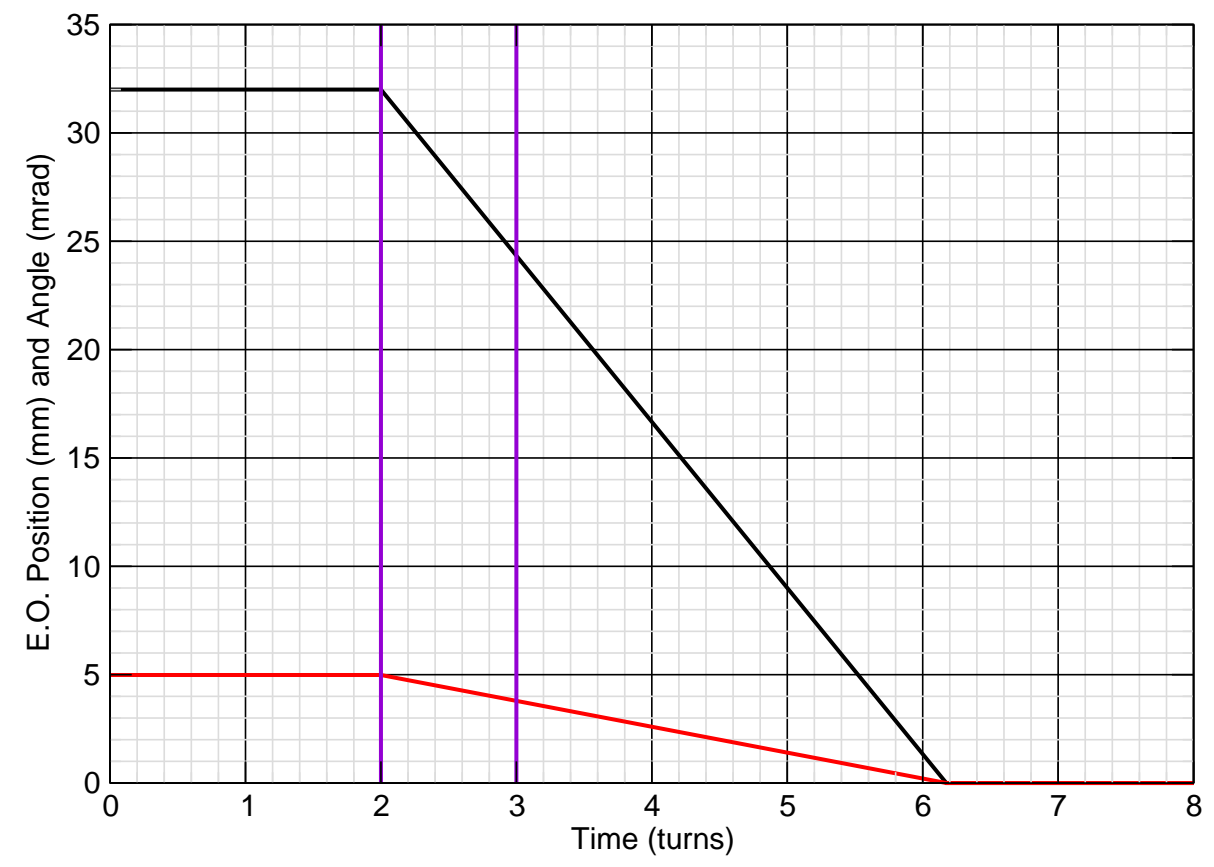

Figure 16: The black and red curves are the injection bump position $X_{c}$ and angle $X_{c}^{\prime}=-\alpha X_{c} / \beta$ as functions of time for one-turn injection of EBIS beams. The time axis is marked in turns, where one turn is $10.3 \mu \mathrm{s}$. The beam pulse occurs between the two violet lines. The horizontal and vertical tunes are $Q_{H}=4.70$ and $Q_{V}=4.81$. The MAD program gives lattice parameters $\alpha=-1.729$ and $\beta=11.101 \mathrm{~m}$ at the inflector exit. The magnetic rigidity is taken to be $1.255 \mathrm{Tm}$ which is the expected maximum for EBIS beams. 


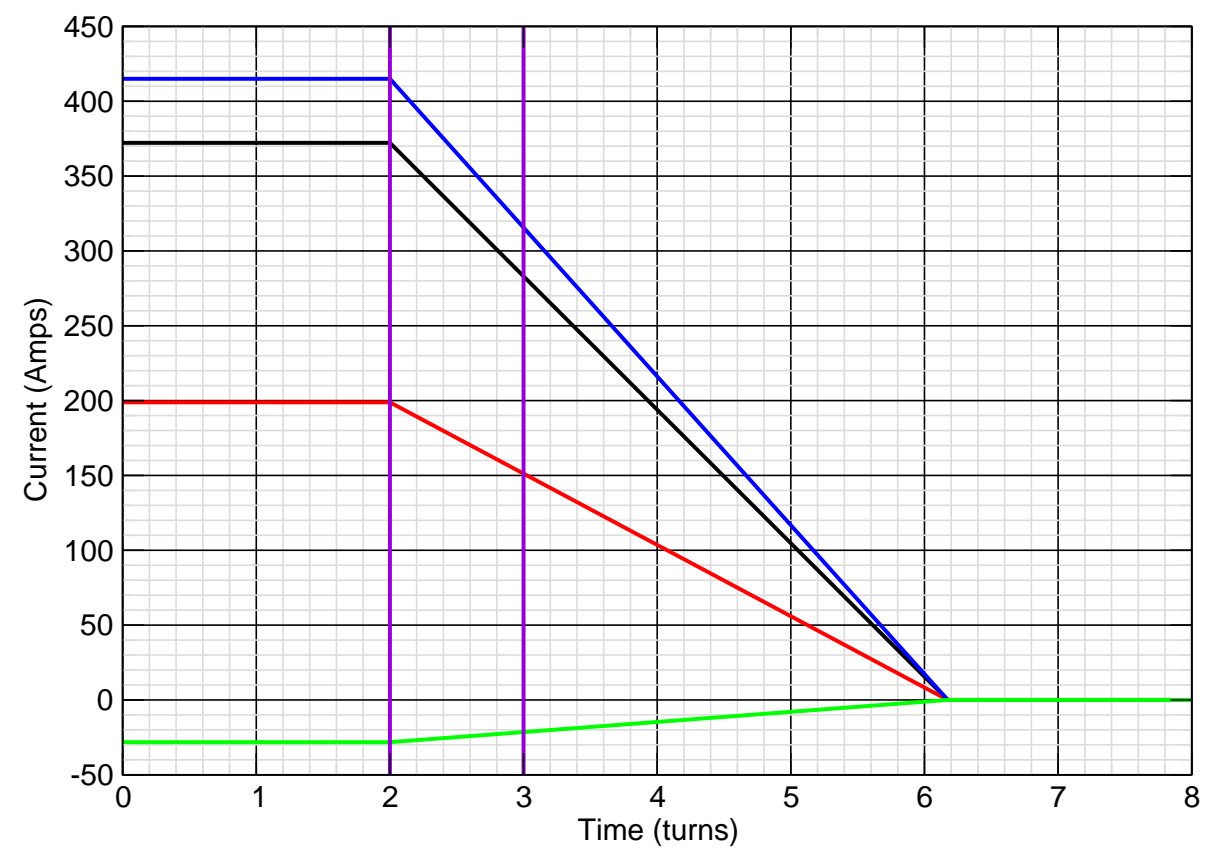

Figure 17: The black, red, blue, and green curves are the C1, C3, C7, and D1 dipole currents, respectively, for the injection bump function of Figure 16. The one-turn EBIS beam pulse occurs between the two violet lines. The rate of fall of the $\mathrm{C} 7$ dipole current (blue curve) is $9.651 \mathrm{~A} / \mu \mathrm{s}$. 


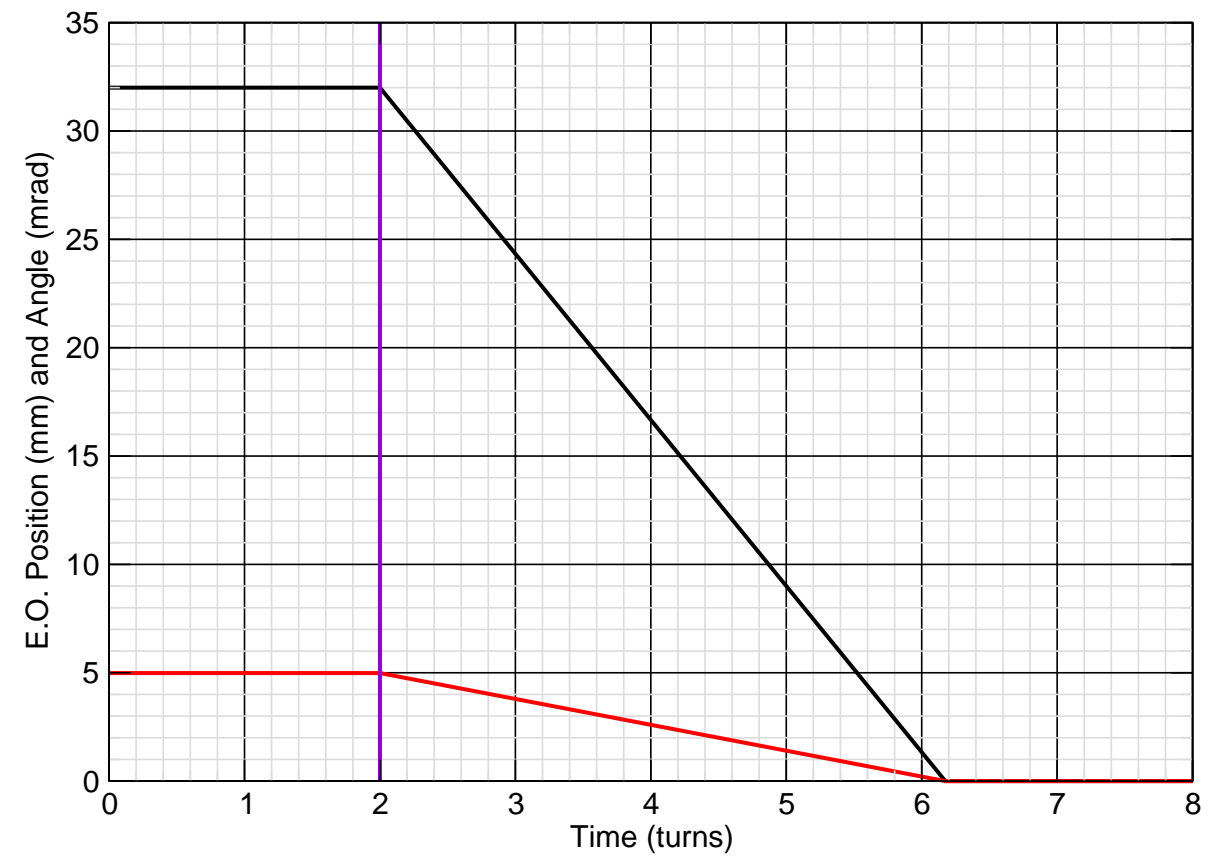

Figure 18: We consider the evolution of a beam ellipse launched from the injection point (i.e. the exit of the $\mathrm{C} 3$ inflector) at time $\mathbf{2 . 0}$ turns as indicated by the violet line above. Figures $\mathbf{1 9}$ and $\mathbf{2 0}$ show the evolution of the beam ellipse. The necessary computations were carried out with Fortran program "EBinject11" using MAD Twiss file TwissQH47QV481. 


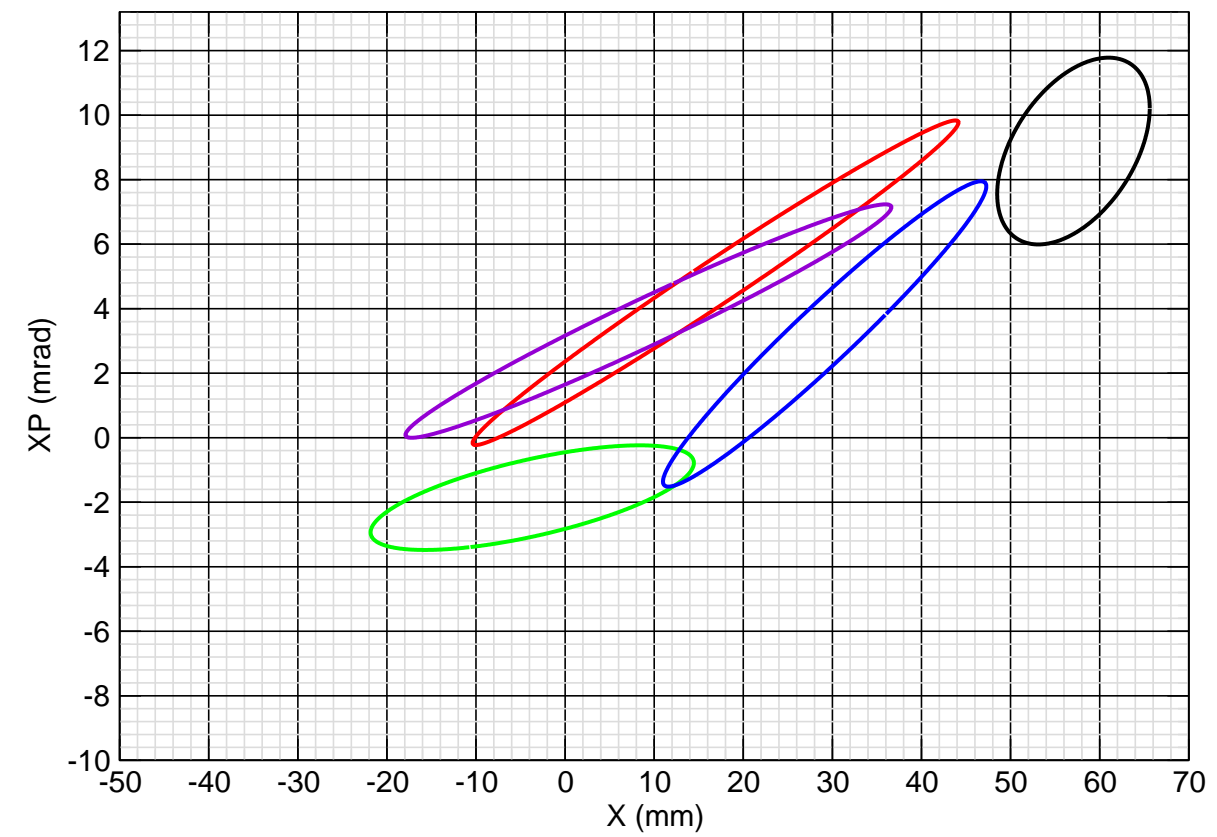

Figure 19: The black, red, green, blue, and violet curves are the beam ellipse after $0,1,2,3$, and 4 turns, respectively, from launch time 2.0 turns. The initial (black) ellipse has parameter $B=0.3$ and area $\pi \epsilon_{0}=22.0 \pi \mathrm{mm}$ milliradians. The outer side of the $1 \mathrm{~mm}$ thick inflector septum is located at $X=48.5 \mathrm{~mm}$. The tunes are $Q_{H}=4.70$ and $Q_{V}=4.81$. 


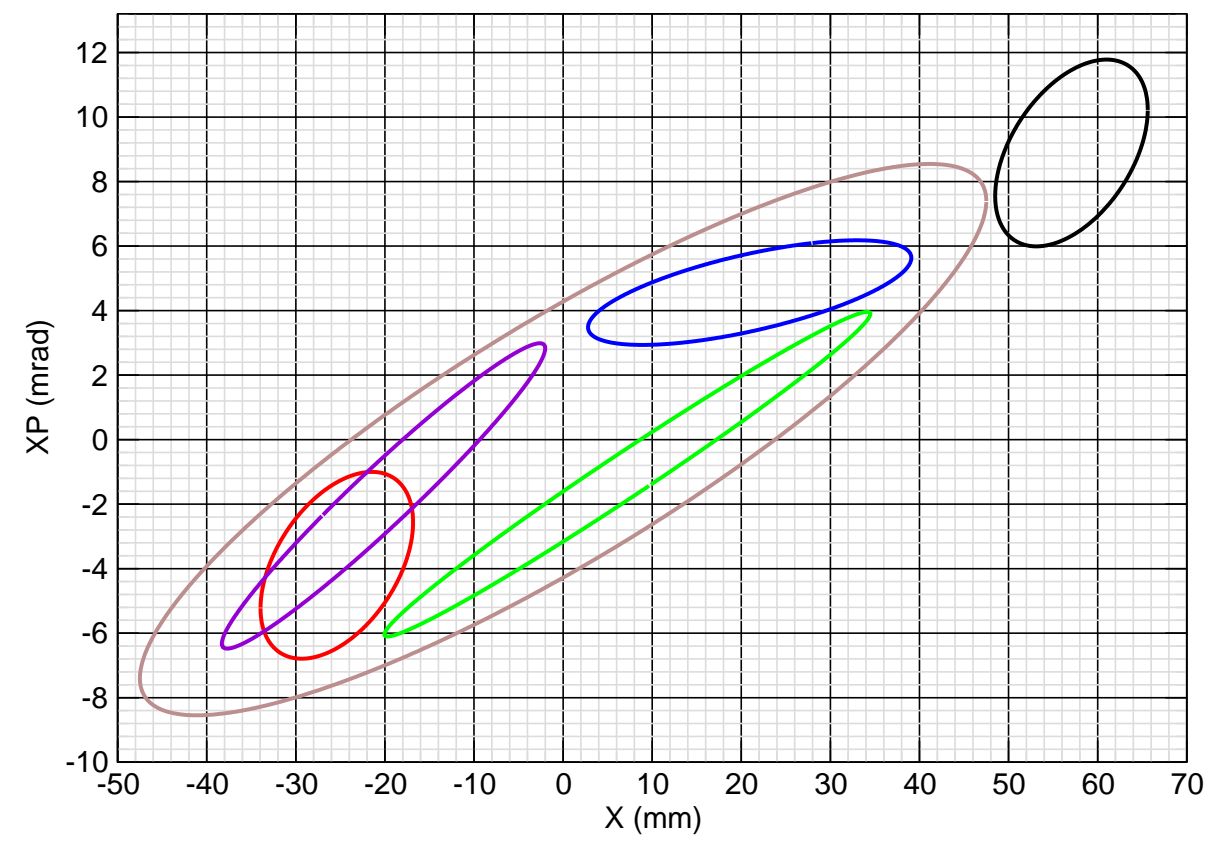

Figure 20: The black, red, green, blue, and violet curves are the beam ellipse after $0,5,6,7$, and 8 turns, respectively, from launch time 2.0 turns. The large brown ellipse is the machine ellipse that just touches the inflector septum. Its area is $203.249 \pi \mathrm{mm}$ milliradians which may be taken to be the acceptance of the machine. The initial (black) ellipse launched at time 2.0 turns therefore ends up inside the machine acceptance after the bump has collapsed. 


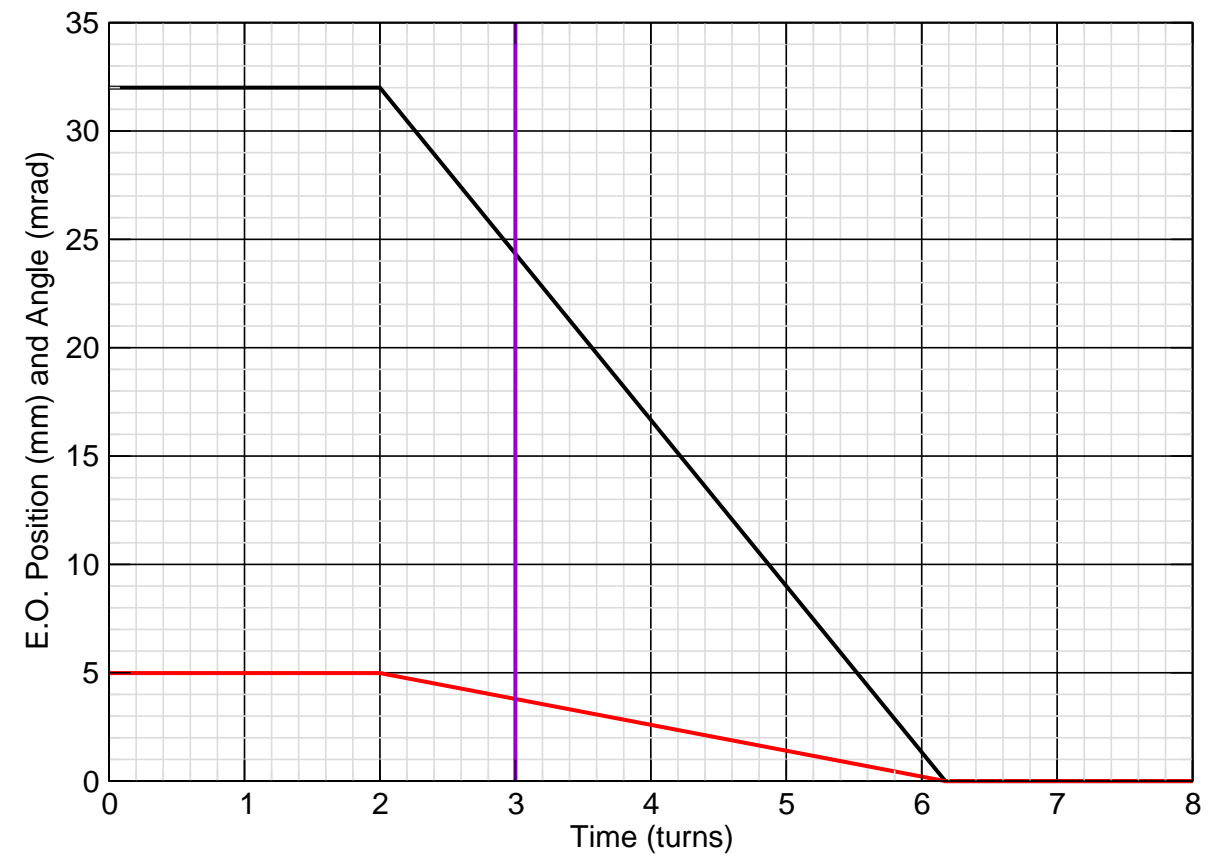

Figure 21: Now consider the evolution of a beam ellipse launched from the injection point (i.e. the exit of the $\mathrm{C} 3$ inflector) at time 3.0 turns as indicated by the violet line above. Figures $\mathbf{2 2}$ and $\mathbf{2 3}$ show the evolution of the beam ellipse. The necessary computations were carried out with Fortran program "EBinject11" using MAD Twiss file TwissQH47QV481. 


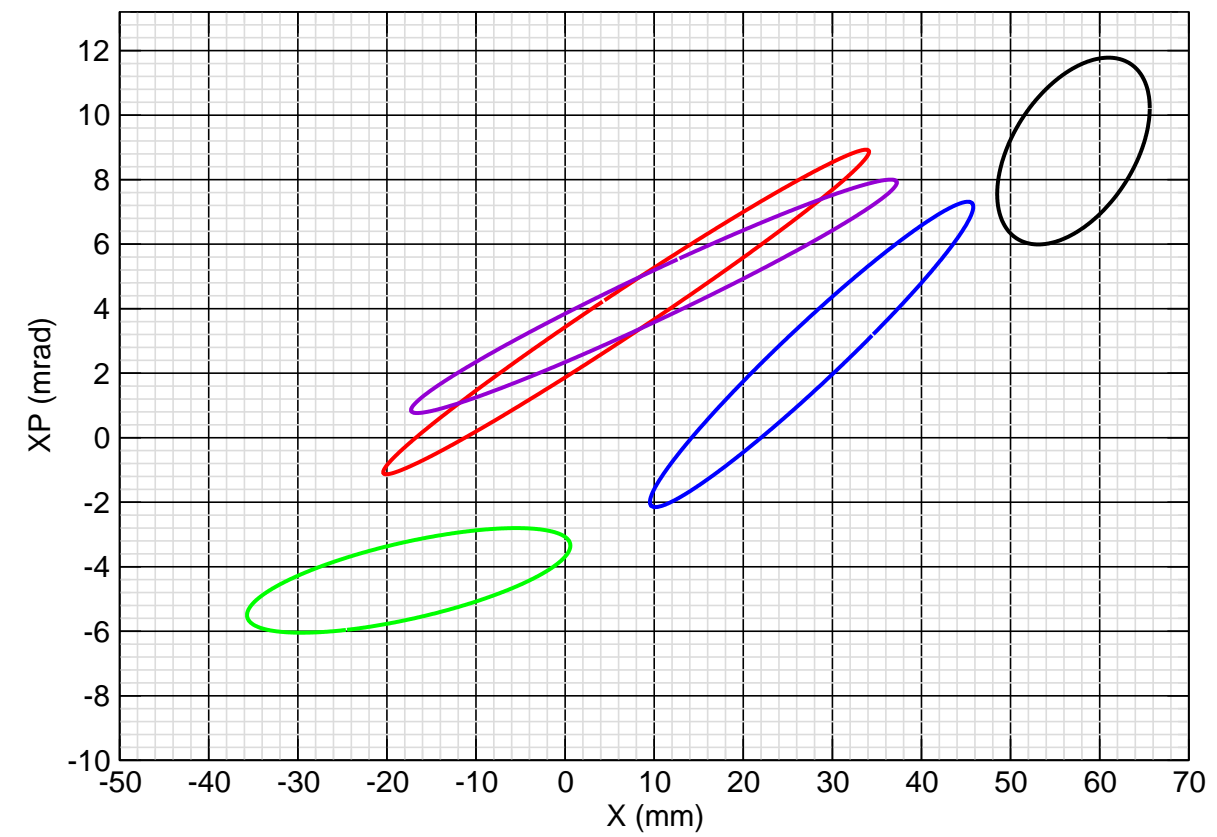

Figure 22: The black, red, green, blue, and violet curves are beam ellipse after $0,1,2,3$, and 4 turns, respectively, from launch time 3.0 turns. The initial (black) ellipse has parameter $B=0.3$ and area $\pi \epsilon_{0}=22.0 \pi \mathrm{mm}$ milliradians. The outer side of the $1 \mathrm{~mm}$ thick inflector septum is located at $X=48.5 \mathrm{~mm}$. The tunes are $Q_{H}=4.70$ and $Q_{V}=4.81$. 


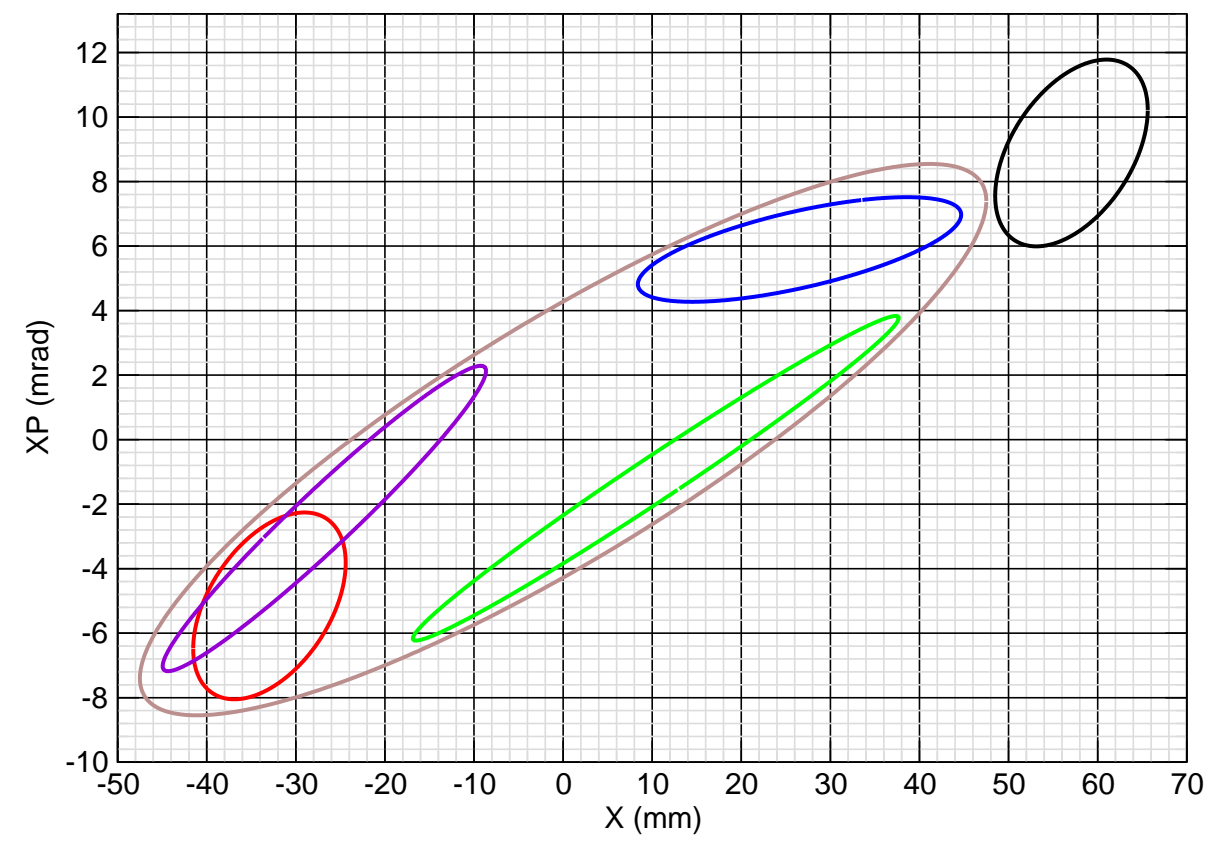

Figure 23: The black, red, green, blue, and violet curves are the beam ellipse after $0,5,6,7$, and 8 turns, respectively, from launch time 3.0 turns. Here again the large brown ellipse is the machine ellipse that just touches the inflector septum. Its area is $203.249 \pi \mathrm{mm}$ milliradians which may be taken to be the acceptance of the machine. The initial (black) ellipse launched at time 3.0 turns therefore ends up inside the machine acceptance after the bump has collapsed. 


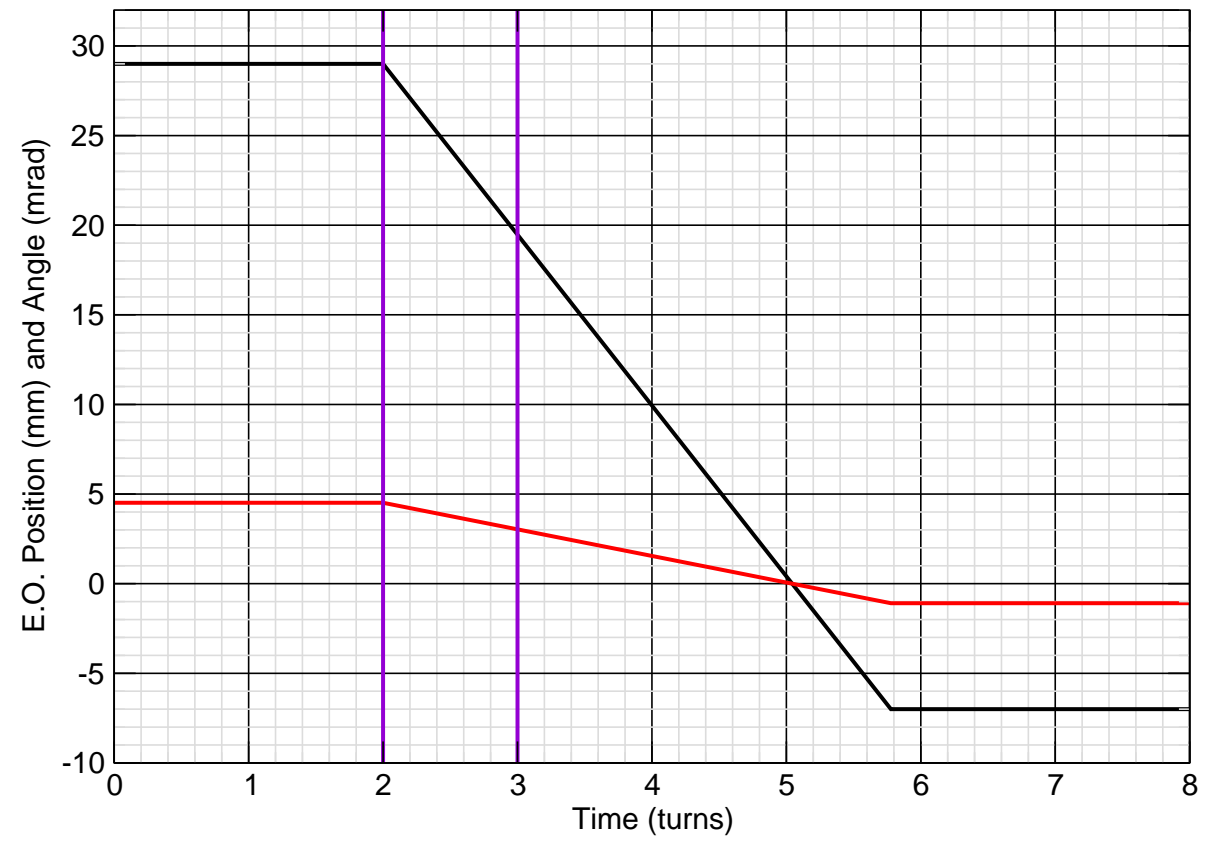

Figure 24: The black and red curves are the injection bump position $X_{c}$ and angle $X_{c}^{\prime}=-\alpha_{I} X_{c} / \beta_{I}$ as functions of time for one-turn injection of EBIS beams. The time axis is marked in turns, where one turn is 10.3 $\mu \mathrm{s}$. The beam pulse occurs between the two violet lines. The horizontal and vertical tunes are $Q_{H}=4.70$ and $Q_{V}=4.81$. The MAD program gives $\alpha_{I}=-1.729$ and $\beta_{I}=11.101 \mathrm{~m}$. The magnetic rigidity is taken to be $1.255 \mathrm{Tm}$ which is the expected maximum for EBIS beams. Note that the closed orbit has been offset so that it is $7 \mathrm{~mm}$ to the inside of the beam pipe centerline when the current in the injection dipoles is zero. This is needed to keep the beam ellipse launched at the end of the beam pulse away from the septum. 


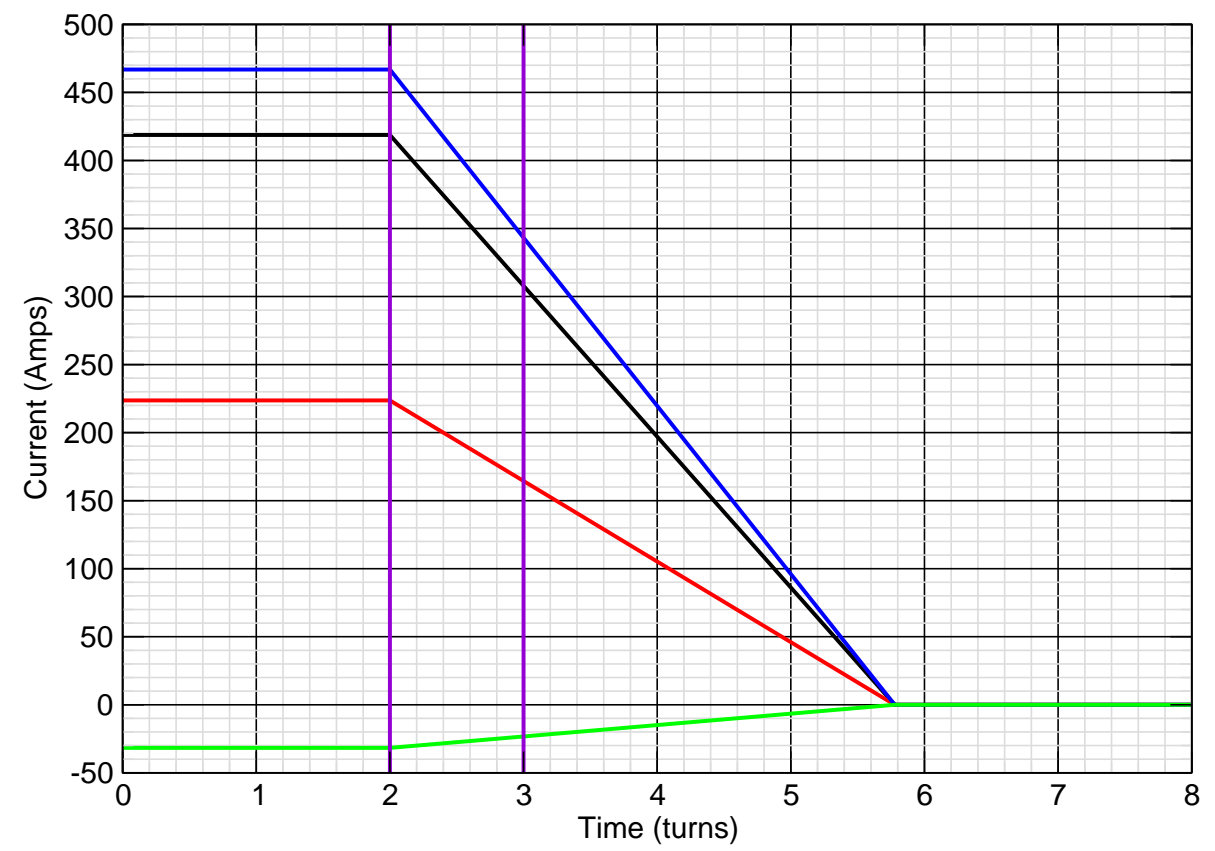

Figure 25: The black, red, blue, and green curves are the C1, C3, C7, and D1 dipole currents, respectively, for the injection bump function of Figure 24. The one-turn EBIS beam pulse occurs between the two violet lines. The rate of fall of the $\mathrm{C} 7$ dipole current (blue curve) is $12.0 \mathrm{~A} / \mu \mathrm{s}$. 


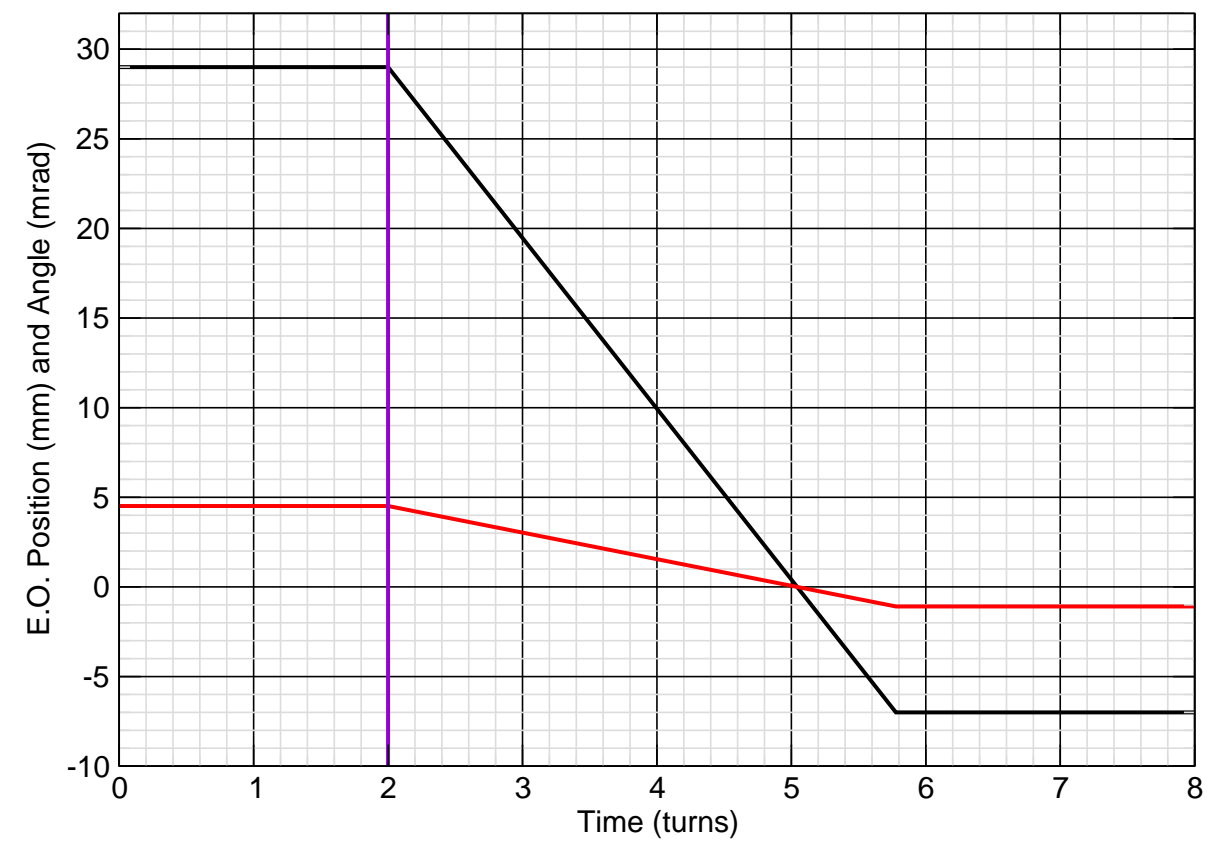

Figure 26: We consider the evolution of a beam ellipse launched from the injection point (i.e. the exit of the $\mathrm{C} 3$ inflector) at time 2.0 turns as indicated by the violet line above. Figures $\mathbf{2 7}$ and $\mathbf{2 8}$ show the evolution of the beam ellipse. The necessary computations were carried out with Fortran program "EBinject12" using MAD Twiss file TwissQH47QV481. 


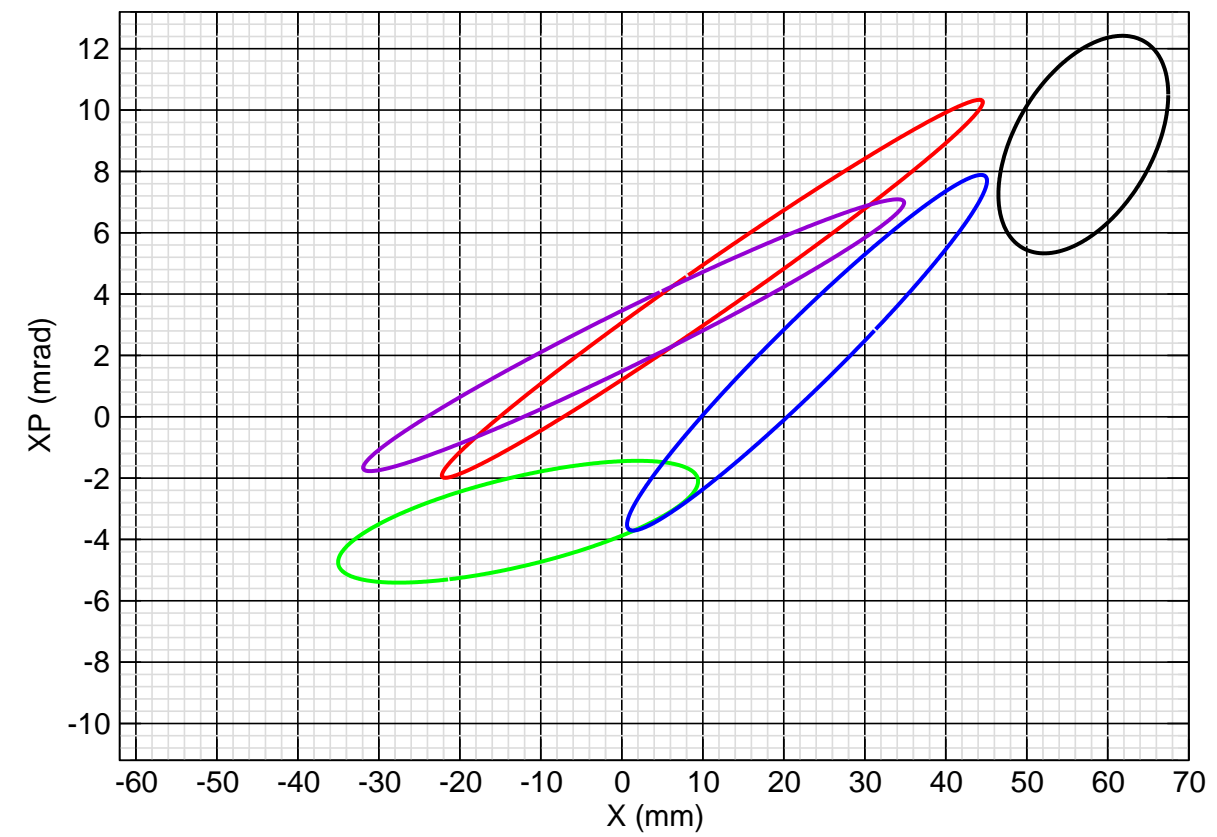

Figure 27: The black, red, green, blue, and violet curves are the beam ellipse after $0,1,2,3$, and 4 turns, respectively, from launch time 2.0 turns. The initial (black) ellipse has parameter $B=0.3$ and area $\pi \epsilon_{0}=33.0 \pi \mathrm{mm}$ milliradians. The outer side of the $1 \mathrm{~mm}$ thick inflector septum is located at $X=46.5 \mathrm{~mm}$. The tunes are $Q_{H}=4.70$ and $Q_{V}=4.81$. 


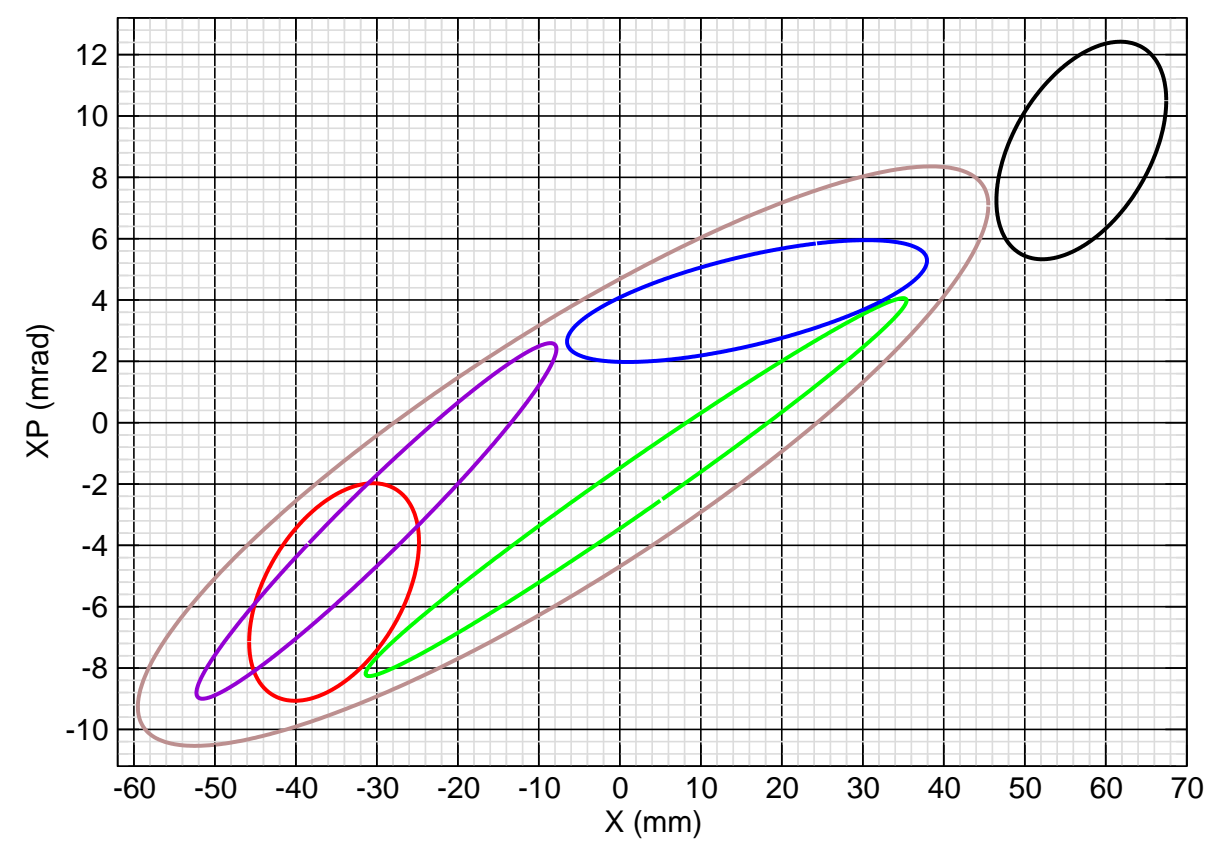

Figure 28: The black, red, green, blue, and violet curves are the beam ellipse after $0,5,6,7$, and 8 turns, respectively, from launch time 2.0 turns. The large brown ellipse is the machine ellipse that just touches the inflector septum. Its area is $248.290 \pi \mathrm{mm}$ milliradians which may be taken to be the acceptance of the machine. The initial (black) ellipse launched at time 2.0 turns therefore ends up inside the machine acceptance after the bump has collapsed. 


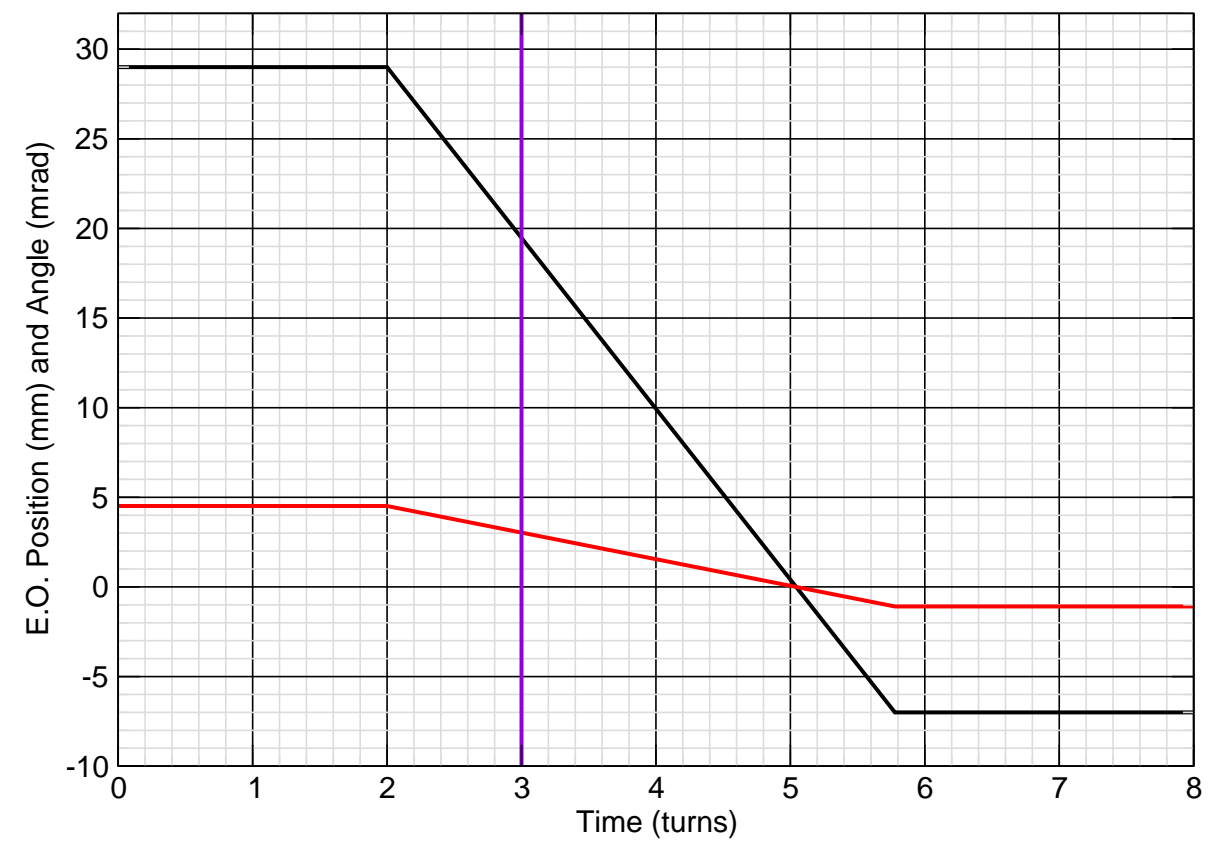

Figure 29: Now consider the evolution of a beam ellipse launched from the injection point (i.e. the exit of the $\mathrm{C} 3$ inflector) at time 3.0 turns as indicated by the violet line above. Figures $\mathbf{3 0}$ and $\mathbf{3 1}$ show the evolution of the beam ellipse. The necessary computations were carried out with Fortran program "EBinject12" using MAD Twiss file TwissQH47QV481. 


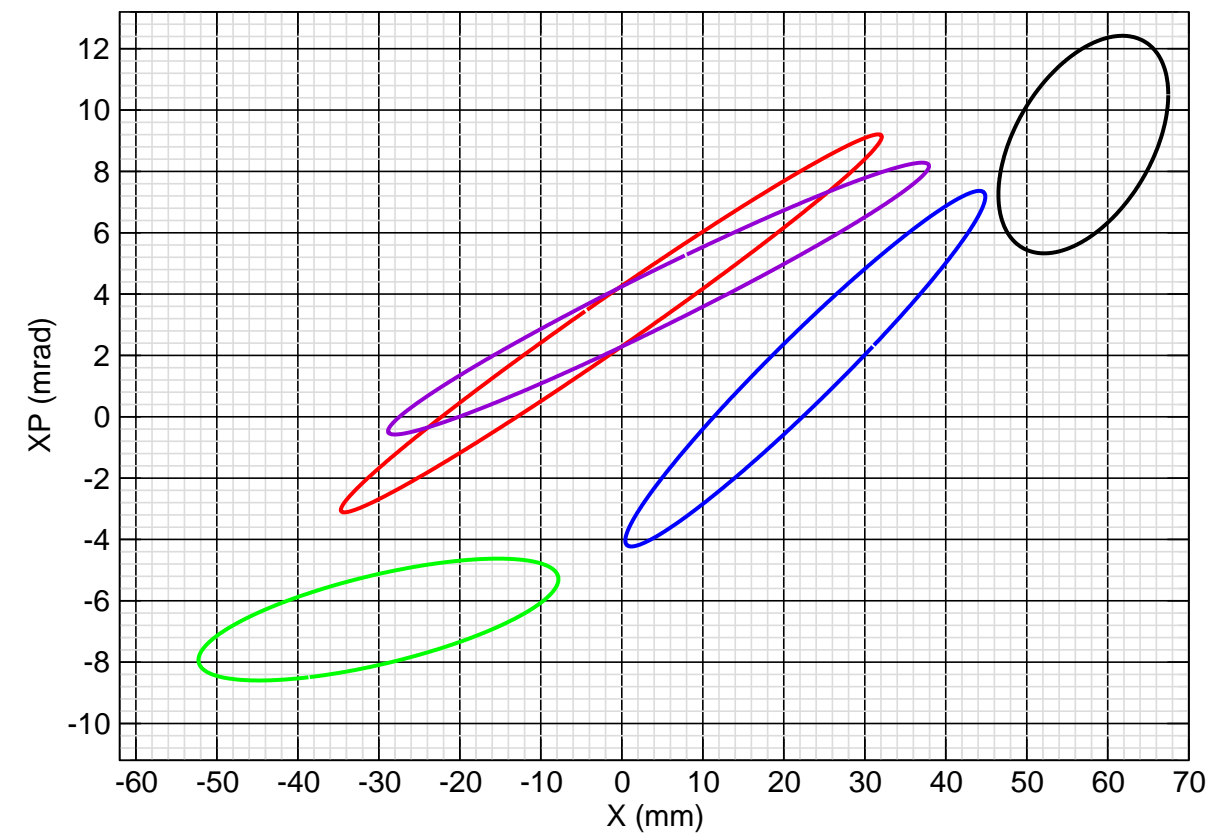

Figure 30: The black, red, green, blue, and violet curves are beam ellipse after $0,1,2,3$, and 4 turns, respectively, from launch time 3.0 turns. The initial (black) ellipse has parameter $B=0.3$ and area $\pi \epsilon_{0}=33.0 \pi \mathrm{mm}$ milliradians. The outer side of the $1 \mathrm{~mm}$ thick inflector septum is located at $X=46.5 \mathrm{~mm}$. The tunes are $Q_{H}=4.70$ and $Q_{V}=4.81$. 


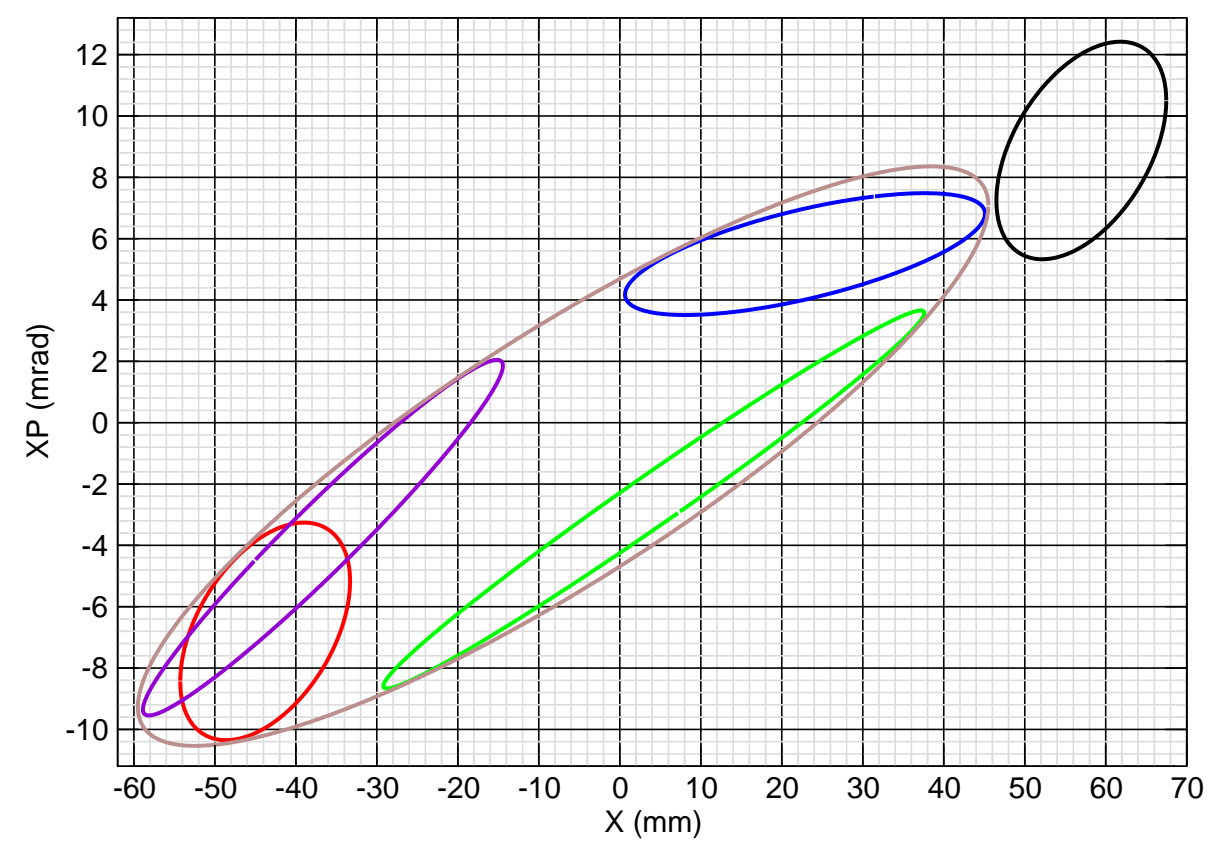

Figure 31: The black, red, green, blue, and violet curves are the beam ellipse after $0,5,6,7$, and 8 turns, respectively, from launch time 3.0 turns. Here again the large brown ellipse is the machine ellipse that just touches the inflector septum. Its area is $248.290 \pi \mathrm{mm}$ milliradians which may be taken to be the acceptance of the machine. The initial (black) ellipse launched at time 3.0 turns therefore ends up inside the machine acceptance after the bump has collapsed. 